\title{
Interaction of an atom with layered dielectrics
}

\author{
Claudia Eberlein and Robert Zietal \\ Department of Physics \& Astronomy, University of Sussex, Falmer, Brighton BN1 9QH, England
}

(Received 10 October 2010; published 7 December 2010)

\begin{abstract}
We determine the energy-level shift experienced by a neutral atom due the quantum electromagnetic interaction with a layered dielectric body. We use the technique of normal-mode expansion to quantize the electromagnetic field in the presence of a layered, nondispersive, and nonabsorptive dielectric. We explicitly calculate the equal-time commutation relations between the electric field and vector potential operators. We show that the commutator can be expressed in terms of a generalized transverse $\delta$ function and that this a consequence of using the generalized Coulomb gauge to quantize the electromagnetic field. These mathematical tools turn out to be very helpful in the calculation of the energy-level shift of the atom, which can be in its ground state or excited. The results for the shift are then analyzed asymptotically in various regions of the system's parameter space, with a view to providing quick estimates of the influence of a single dielectric layer on the Casimir-Polder interaction between an atom and a dielectric half space. We also investigate the impact of resonances between the wavelength of the atomic transition and the thickness of the top layer.
\end{abstract}

DOI: 10.1103/PhysRevA.82.062506

\section{INTRODUCTION}

The question of the interaction between a neutral atom and a macroscopic dielectric body, once of purely academic interest, has recently been promoted to a real-life physics problem thanks to the rapid developments in nanotechnology and experimental techniques. It is no longer the case that this interaction, the so-called Casimir-Polder interaction, is a tiny effect that can be ignored in all practical situations. Instead, on the length scales that nanotechnology nowadays operates in, dispersion forces, as they are also called, become significant and may appreciably influence miniaturized physical systems. Many of the current ambitions of cold-atom physics toward quantum computation and a variety of nanotechnological applications involves the trapping and accurate guiding of single atoms above dielectric substrates, so-called atom chips. With these the nearby environment of a trapped atom usually consists of a complicated array of inhomogeneous dielectrics. The questions then arising are the following: What are the magnitudes of the Casimir-Polder forces felt by the atom? Can one possibly engineer the types and shapes of surrounding materials either to minimize unwanted dispersion forces or to make them optimally contribute to the trapping or guiding? In order to investigate such possibilities one needs to go beyond simple featureless geometries and ground-state atoms and gain flexibility. The perhaps least sophisticated but still interesting example to study in this context is to consider a neutral atom, possibly excited, above a layered dielectric half space (cf. Fig. 1). If the atom is in its ground state, then the Casimir-Polder force is always attractive for material surfaces with refractive indices greater than 1 . In such case it is desirable to derive simple analytical formulas that would allow one to obtain quick estimates of the magnitudes of the forces involved in terms of the optical properties of the layer and the substrate [1]. On the other hand, if the atom is in its excited state, then, as it is widely recognized [2], the potential acquires an oscillatory contribution that can result in a repulsive force. Additionally, the presence of the layer creates the possibility of a resonance between the wavelength of the atomic transition and the thickness of the layer, which could lead to a suppression or enhancement of the interaction.
PACS number(s): 31.70.-f, 41.20.Cv, 42.50.Pq

There exist a variety of theoretical approaches devised to study the Casimir-Polder interaction (see, e.g., [3] for a recent list of references) but perhaps the most successful ones being the linear-response theory [4] and phenomenological macroscopic QED [5]. By using linear-response theory [4] and expressing the field susceptibilities in terms of Fresnel reflection coefficients $[2,6]$, one can express the Casimir-Polder interaction as an integral along the imaginary frequency axis of the product of the atomic and field susceptibilities. Thus, in practice the problem is reduced to the calculation of the classical electromagnetic Green's tensor expressed in terms of Fresnel coefficients. Such calculations, while straightforward in principle, tend to be quite tedious and often inevitably lead to the use of numerical methods. However, there is a benefit to studying problems in quantum electrodynamics by using physically transparent methods that do not obscure the basic underlying physics. For the kind of geometry of plane layered dielectrics considered in this paper, the technique of electromagnetic field quantization based on a normal-mode expansion [7] seems to best emphasize the physics of the problem, namely, the fact that the system supports two kinds of modes of the electromagnetic field [8]: These are traveling modes with a continuous spectrum and trapped modes with a discrete spectrum, that is, occurring at only certain allowed frequencies. The trapped modes arise because of repeated total internal reflections within the top layer of higher refractive index than the substrate and emerge as evanescent waves outside the wave guide. This gives rise to an intricate assortment of evanescent modes outside a layered dielectric where evanescent waves with continuous spectrum, also arising in a half-space geometry [7], are superposed with discrete evanescent modes that arise only in the presence of the slablike wave guide [1]. In the framework in which we apply in this work, in the same spirit as, for example, [1,9], the use of standard perturbation theory renders all calculations explicit and it is possible from the outset to track down and remove, if necessary, any ambiguities that tend to remain hidden in more elaborate theories. For example, linear-response theory results in an integral over the Fresnel reflection coefficients but gives 


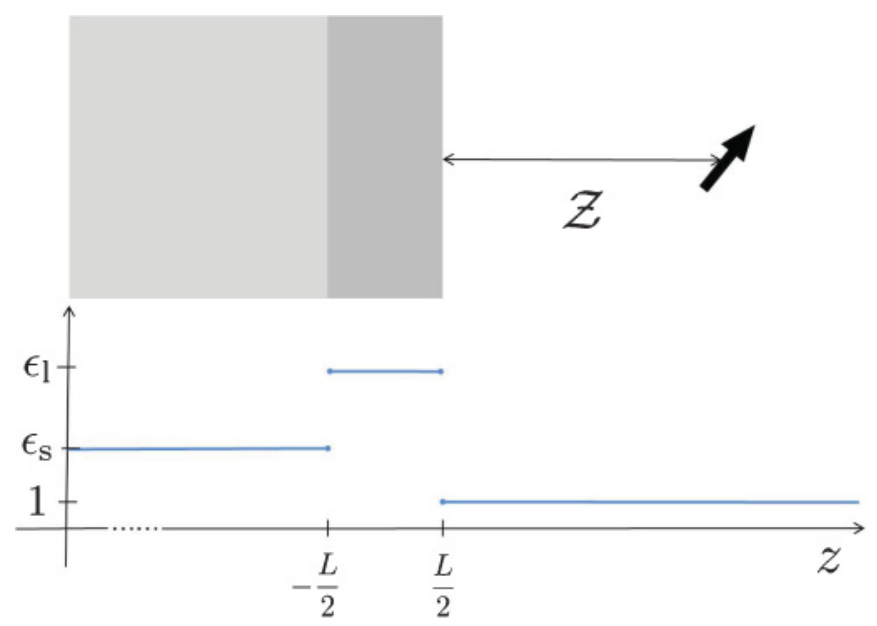

FIG. 1. (Color online) Atomic dipole moment in the vicinity of the layered dielectric. The dielectric function is a piecewise constant function of the coordinate $z$.

no indication of whether the evanescent waves associated with the trapped modes contribute to the Casimir-Polder interaction or not. The question is answered at once if the normal-modes approach is used instead (see [8,10]). Also, interpretations of more complicated field-theoretical approaches [11] can be put to an explicit test [1].

The purpose of this paper is twofold. First, it aims to support current experimental efforts by providing a range of analytical formulas useful for quick estimates of the dispersion forces acting on an atom placed in the vicinity of the layered dielectric, with particular emphasis on the corrections caused by the layer as compared to the standard half-space results reported in [9]. It also investigates the resonant interaction between an excited atom and a layer in the search for the possible enhancement or suppression of the Casimir-Polder force. Second, it formulates a simple and explicit theory based on well-understood concepts of theoretical physics such as perturbation theory and electromagnetic field quantization in terms of a normal-modes expansion. The theoretical aspect, although serving only as a means to a practical end result, turns out to be interesting in its own right. The perturbative approach used in this work leads to the problem of the summation over the modes of the electromagnetic field, which is nontrivial because of the dual character of the modes of the electromagnetic field. The task of adding the discrete and continuous field modes is elegantly accomplished by the use of complex-integration techniques. This allows us to explicitly show that the canonical commutation relations between the field operators are satisfied, which is equivalent to saying that the completeness relation of the normal-modes holds in the geometry considered. Although this is not a surprise because the field modes are solutions of a Hermitian operator's eigenvalue problem, the explicit calculation we carry out provides us with the mathematics necessary to complete a typical perturbative calculation in this geometry. It also allows us to cast the end result in a simple and elegant form that is easy to study analytically in various asymptotic regimes. The same technique could be applied to any similar perturbative calculation in such a geometry.
This paper is organized as follows. First we quantize the electromagnetic field in the presence of a layered dielectric (Sec. II). Then, in Sec. II C, we explicitly prove the completeness relation for the electromagnetic field modes. Equipped with the necessary mathematical tools, we proceed to calculate the energy shift in Sec. III and then study it analytically (Sec. IV) and numerically (Sec. V).

\section{FIELD QUANTIZATION IN THE PRESENCE OF A LAYERED BOUNDARY}

Our ultimate aim is to work out the energy-level shift in an atom caused by the presence of a layered dielectric. In order to obtain a result that fully takes into account retardation effects, the quantization of the electromagnetic field is necessary. To emphasize the physics of the problem we choose to quantize the electromagnetic field using a normal-mode expansion as described in [12]. The dielectric environment we consider (cf. Fig. 1) consists of a substrate, a dielectric half-space occupying the region of space $z<-L / 2$ described by a dielectric constant $\epsilon_{\mathrm{s}}=n_{\mathrm{s}}^{2}$, and on top of that substrate an additional dielectric layer of thickness $L$, which has a dielectric constant $\epsilon_{1}=n_{1}^{2}$. We assume that the dielectric constant of the layer is higher than that of the substrate $\epsilon_{1}>\epsilon_{\mathrm{S}}$ in order to account for modes that are trapped inside the layer. Although we work with this assumption, the final result will turn out to be valid even when the reflectivity of the substrate exceeds that of the layer, but that is the physically less interesting case. Throughout this paper we assume all dielectric constants to be frequency independent so that the optical properties of the system are described solely by a pair of real numbers, $\epsilon_{1}$ and $\epsilon_{\mathrm{s}}$.

To solve Maxwell equations for the electromagnetic field operators in the Heisenberg's picture we introduce, in the usual manner [13], the electromagnetic potentials $\mathbf{A}(\mathbf{r}, t)$ and $\Phi(\mathbf{r}, t)$ and work in the generalized Coulomb gauge

$$
\nabla \cdot[\epsilon(\mathbf{r}) \mathbf{A}(\mathbf{r})]=0,
$$

with the dielectric permittivity being a piecewise constant function as shown in Fig. 1. In the absence of free charges one can set $\Phi(\mathbf{r}, t)=0$ and work only with the vector potential $\mathbf{A}(\mathbf{r}, t)$ which satisfies the wave equation

$$
\nabla^{2} \mathbf{A}(\mathbf{r}, t)-\epsilon(z) \frac{\partial^{2}}{\partial t^{2}} \mathbf{A}(\mathbf{r}, t)=0, \quad|z| \neq L / 2 .
$$

Note that right on the interfaces condition (1) is singular due to discontinuities of the dielectric function and Eq. (2) does not hold at these points. The normal modes of the field $\mathbf{f}(\mathbf{r}) e^{i \omega t}$ satisfy the Helmholtz equation

$$
\nabla^{2} \mathbf{f}_{\mathbf{k} \lambda}(\mathbf{r})+\epsilon(z) \omega^{2} \mathbf{f}_{\mathbf{k} \lambda}(\mathbf{r})=0, \quad|z| \neq L / 2
$$

and we have labeled them by their wave vector $\mathbf{k}$ and polarization $\lambda=\{\mathrm{TE}, \mathrm{TM}\}$. This mode decomposition allows one to solve the field equation (2) in each distinct region of space separately and then stitch up the solutions across the interfaces by demanding that they are consistent with the Maxwell boundary conditions, that is, that $\mathbf{E}_{\|}, D_{\perp}$, and $\mathbf{B}$ are all continuous. 
The Helmholtz equation (3) is, in fact, the eigenvalue problem of a Hermitian operator [12]

$$
\left[\frac{1}{\sqrt{\epsilon}} \nabla \times \nabla \times \frac{1}{\sqrt{\epsilon}}\right] \sqrt{\epsilon} \mathbf{f}_{\mathbf{k} \lambda}(\mathbf{r})=-\omega^{2} \sqrt{\epsilon} \mathbf{f}_{\mathbf{k} \lambda}(\mathbf{r}),
$$

so that we expect the field modes $\sqrt{\epsilon} \mathbf{f}_{\mathbf{k} \lambda}(\mathbf{r})$ to form a complete set of functions suitable for describing any field configuration. The completeness relation takes the form

$$
\sum_{\lambda} \int d^{2} \mathbf{k}_{\|} \sum_{k_{z}} f_{\mathbf{k} \lambda}^{i}(\mathbf{r}) f_{\mathbf{k} \lambda}^{* j}\left(\mathbf{r}^{\prime}\right)=\delta_{i j}^{\epsilon}\left(\mathbf{r}, \mathbf{r}^{\prime}\right), \quad z, z^{\prime}>\frac{L}{2},
$$

with $\delta_{i j}^{\epsilon}\left(\mathbf{r}, \mathbf{r}^{\prime}\right)$ being the unit kernel in the subspace of functions satisfying (1); we call this the generalized transverse $\delta$ function. From quite general considerations [14] we can expect it to be given by

$$
\delta_{i j}^{\epsilon}\left(\mathbf{r}, \mathbf{r}^{\prime}\right)=\delta_{i j} \delta^{(3)}\left(\mathbf{r}-\mathbf{r}^{\prime}\right)-\nabla_{i} \nabla_{j}^{\prime} G\left(\mathbf{r}, \mathbf{r}^{\prime}\right),
$$

with the electrostatic Green's function of the Laplace equation given by

$$
\begin{aligned}
G\left(\mathbf{r}, \mathbf{r}^{\prime}\right)= & \frac{1}{4 \pi} \frac{1}{\left|\mathbf{r}-\mathbf{r}^{\prime}\right|}-\frac{1}{4 \pi} \int_{0}^{\infty} d k J_{0}(k \rho) e^{-k\left(z+z^{\prime}\right)} \\
& \times \frac{\frac{n_{1}^{2}-1}{n_{1}^{2}+1}-\frac{n_{1}^{2}-n_{\mathrm{s}}^{2}}{n_{\mathrm{s}}^{2}+n_{1}^{2}} e^{-2 k L}}{1-\frac{n_{1}^{2}-1}{n_{1}^{2}+1} \frac{n_{1}^{2}-n_{\mathrm{s}}^{2}}{n_{\mathrm{s}}^{2}+n_{1}^{2}} e^{-2 k L}},
\end{aligned}
$$

where $\rho=\left|\mathbf{r}_{\|}-\mathbf{r}_{\|}^{\prime}\right|$ and, for brevity, we have chosen to confine ourselves to the case $z, z^{\prime}>L / 2$. The function $J_{0}$ in the preceding equation is a Bessel function of the first kind [15], Eq. (9.1.1). The outline of the derivation of the Green's function is given in Appendix B.

The sum over all modes in Eq. (5) is complicated because the spectrum of the field modes has nontrivial structure. It has been shown previously $[8,16]$ that the system supports two kinds of quite distinct types of modes. There are traveling modes going from left to right or in the opposite direction, and there are guided modes that are trapped by the dielectric layer, which essentially acts as a wave guide. The spectrum of the traveling modes is continuous, whereas the spectrum of the modes trapped in the dielectric layer is discrete and only some values of the (perpendicular) wave vector are allowed, namely, those satisfying a certain dispersion relation. This dual character of the spectrum of the field modes is a major obstacle in working with these modes and calculating, for example, the energy shift of an atom nearby, but an elegant solution to this problem has been developed in [17], whose basic idea we follow here.

We choose the normalization of the mode functions $\sqrt{\epsilon} \mathbf{f}_{\mathbf{k} \lambda}(\mathbf{r})$ according to the convention

$$
\begin{aligned}
& \int d^{3} \mathbf{r} \epsilon(z) \mathbf{f}_{\mathbf{k} \lambda}^{*}(\mathbf{r}) \cdot \mathbf{f}_{\mathbf{k}^{\prime} \lambda^{\prime}}(\mathbf{r}) \\
&=\left\{\begin{array}{cc}
\delta_{\lambda \lambda^{\prime}} \delta^{(3)}\left(\mathbf{k}-\mathbf{k}^{\prime}\right) & \text { (traveling modes), } \\
\delta_{\lambda \lambda^{\prime}} \delta^{(2)}\left(\mathbf{k}_{\|}-\mathbf{k}_{\|}^{\prime}\right) \delta_{k_{z} k_{z}^{\prime}} & \text { (trapped modes). }
\end{array}\right.
\end{aligned}
$$

Then, the electric field $\mathbf{E}(\mathbf{r})=-\partial_{t} \mathbf{A}(\mathbf{r})$ expanded in terms of the normal modes can be written as

$$
\mathbf{E}(\mathbf{r})=i \sum_{\lambda} \int d^{2} \mathbf{k}_{\|} \sum_{k_{z}} \sqrt{\frac{\omega_{\mathbf{k}}}{2 \epsilon_{0}}} a_{\mathbf{k} \lambda} \mathbf{f}_{\mathbf{k} \lambda}(\mathbf{r}) e^{-i \omega_{\mathbf{k}} t}+\text { H.c. }
$$

where H.c. stands for Hermitian conjugate. The photon creation and annihilation operators, $a_{\mathbf{k} \lambda}^{\dagger}$ and $a_{\mathbf{k} \lambda}$, satisfy bosonic commutation relation

$$
\left[a_{\mathbf{k} \lambda}, a_{\mathbf{k} \lambda^{\prime}}^{\dagger}\right]=\delta_{\lambda \lambda^{\prime}}\left\{\begin{array}{l}
\delta^{(3)}\left(\mathbf{k}-\mathbf{k}^{\prime}\right), \\
\delta^{(2)}\left(\mathbf{k}_{\|}-\mathbf{k}_{\|}^{\prime}\right) \delta_{k_{z} k_{z}^{\prime}},
\end{array}\right.
$$

where the top and bottom of the right-hand side corresponds to the traveling and trapped photons, respectively. In order to be able to write out the electromagnetic field operators explicitly one needs to solve the eigenvalue problem (3) and determine the spatial dependence of functions $\mathbf{f}_{\mathbf{k} \lambda}(\mathbf{r})$, so we turn our attention to this now.

\section{A. Traveling modes}

Before we work out the traveling modes, for further convenience, we introduce Fresnel coefficients for a single interface. For that we assume that a plane wave is traveling from a medium with refractive index $n_{\mathrm{b}}$ to a medium with the refractive index $n_{\mathrm{a}}$ and that the interface is the $z=0$ plane. Then the standard Fresnel reflection and transmission coefficients are given by [13]

$$
\begin{gathered}
r_{\mathrm{TE}}^{\mathrm{ba}}=\frac{k_{z \mathrm{~b}}-k_{z \mathrm{a}}}{k_{z \mathrm{~b}}+k_{z \mathrm{a}}}, \quad t_{\mathrm{TE}}^{z \mathrm{a}}=\frac{2 k_{z \mathrm{~b}}}{k_{z \mathrm{~b}}+k_{z \mathrm{a}}}, \\
r_{\mathrm{TM}}^{\mathrm{ba}}=\frac{k_{z \mathrm{~b}} / n_{\mathrm{b}}^{2}-k_{z \mathrm{a}} / n_{\mathrm{a}}^{2}}{k_{z \mathrm{~b}} / n_{\mathrm{b}}^{2}+k_{z \mathrm{a}} / n_{\mathrm{a}}^{2}}, \quad t_{\mathrm{TM}}^{z \mathrm{a}}=\frac{2 k_{z \mathrm{~b}} / n_{\mathrm{a}} n_{\mathrm{b}}}{k_{z \mathrm{~b}} / n_{\mathrm{b}}^{2}+k_{z \mathrm{a}} / n_{\mathrm{a}}^{2}},
\end{gathered}
$$

where $k_{z i}$ are the components of the wave vectors perpendicular to the interface in the medium $i=\{\mathrm{a}, \mathrm{b}\}$.

The geometry of the problem (cf. Fig. 1) naturally divides the space into three distinct regions. Consequently, there are three wave vectors to be distinguished. The wave vector in vacuum $(z>L / 2)$,

$$
\mathbf{k}^{ \pm}=\left(k_{x}, k_{y}, \pm k_{z}\right)=\left(\mathbf{k}_{\|}, \pm k_{z}\right),
$$

the wave vector in the dielectric layer $(|z|<L / 2)$,

$$
\mathbf{k}_{1}^{ \pm}=\left(k_{x}, k_{y}, \pm k_{z 1}\right)=\left(\mathbf{k}_{\|}, \pm k_{z 1}\right),
$$

and the wave vector in the substrate $(z<-L / 2)$,

$$
\mathbf{k}_{\mathrm{s}}^{ \pm}=\left(k_{x}, k_{y}, \pm k_{z \mathrm{~s}}\right)=\left(\mathbf{k}_{\|}, \pm k_{z \mathrm{~s}}\right) .
$$

The components of the wave vector that are parallel to the surface are the same for all three regions of space. This follows directly from the requirement that the boundary conditions must be satisfied at all points of a given surface; that is, the spatial phase factors $e^{i \mathbf{k}_{i} \cdot \mathbf{r}}$ must be equal at $z= \pm L / 2$ for all $\mathbf{r}_{\|}$. The different signs of the $z$ components of the wave vectors correspond to the waves propagating in different directions. However, the direction of the propagation of a particular mode needs to be consistent in all three layers so we require that on the real axis

$$
\operatorname{sgn}\left(k_{z}\right)=\operatorname{sgn}\left(k_{z 1}\right)=\operatorname{sgn}\left(k_{z \mathrm{~s}}\right) .
$$

Since the frequency $\omega$ of a single mode is fixed, the $z$ components of the wave vectors in the dielectric are related to the vacuum wave vector $k_{z}$ by

$$
k_{z 1}=\sqrt{\left(n_{1}^{2}-1\right) \mathbf{k}_{\|}^{2}+n_{1}^{2} k_{z}^{2}},
$$




$$
k_{z \mathrm{~s}}=\sqrt{\left(n_{\mathrm{s}}^{2}-1\right) \mathbf{k}_{\|}^{2}+n_{\mathrm{s}}^{2} k_{z}^{2}} .
$$

The mode functions $\mathbf{f}_{\mathbf{k} \lambda}(\mathbf{r})$ are transverse everywhere except right on the interfaces $z= \pm L / 2$ [cf. (1)]. To ensure this transversality, it is convenient to introduce orthonormal polarization vectors,

$$
\mathbf{f}_{\mathbf{k} \lambda}(\mathbf{r})=\hat{\mathbf{e}}_{\lambda}(\mathbf{k}) f_{\mathbf{k} \lambda}(\mathbf{r}),
$$

defined as

$$
\begin{gathered}
\hat{\mathbf{e}}_{\mathrm{TE}}(\nabla)=\left(-\Delta_{\|}\right)^{-1 / 2}\left(-i \nabla_{y}, i \nabla_{x}, 0\right), \\
\hat{\mathbf{e}}_{\mathrm{TM}}(\nabla)=\left(\Delta_{\|} \Delta\right)^{-1 / 2}\left(-\nabla_{x} \nabla_{z},-\nabla_{y} \nabla_{z}, \Delta_{\|}\right),
\end{gathered}
$$

with $\Delta$ being the Laplace operator expressed in Cartesian coordinates and it is understood that the preceding operators act on the factors of the type $e^{i \mathbf{k}_{1}^{ \pm} \mathbf{r}}$, that is, $\hat{\mathbf{e}}_{\lambda}\left(\mathbf{k}_{1}^{ \pm}\right) \equiv \hat{\mathbf{e}}_{\lambda}(\nabla) e^{i \mathbf{k}_{1}^{ \pm} \mathbf{r}}$. Polarization vectors defined in such a way are normalized to unity provided all three components of the wave vector are real. However, they are not of unit length in the case of evanescent waves which have wave vectors with pure imaginary components. The spatial dependence of the mode functions is worked out requiring that each mode consists of the incoming, reflected, and transmitted parts that are joined together by standard boundary conditions across the interfaces, that is, that $\mathbf{E}_{\|}, D_{\perp}$, and $\mathbf{B}$ are continuous. From this it is straightforward to derive that the traveling modes of the system incident from the left, normalized according to (8), are given by

$$
\mathbf{f}_{\mathbf{k} \lambda}^{L}(\mathbf{r})=\frac{\hat{\mathbf{e}}_{\lambda}(\nabla)}{(2 \pi)^{\frac{3}{2}} n_{\mathrm{s}}}\left\{\begin{array}{lc}
e^{i \mathbf{k}_{\mathrm{s}}^{+} \cdot \mathbf{r}}+R_{\lambda}^{L} e^{i \mathbf{k}_{\mathrm{s}}^{-} \cdot \mathbf{r}}, & z<-L / 2, \\
I_{\lambda}^{L} e^{i \mathbf{k}_{1}^{+} \cdot \mathbf{r}}+J_{\lambda}^{L} e^{i \mathbf{k}_{1}^{-} \cdot \mathbf{r}}, & |z|<L / 2, \\
T_{\lambda}^{L} e^{i \mathbf{k}^{+} \cdot \mathbf{r}}, & z>L / 2,
\end{array}\right.
$$

whereas the right-incident modes are given by

$$
\mathbf{f}_{\mathbf{k} \lambda}^{R}(\mathbf{r})=\frac{\hat{\mathbf{e}}_{\lambda}(\nabla)}{(2 \pi)^{\frac{3}{2}}}\left\{\begin{array}{lc}
T_{\lambda}^{R} e^{i \mathbf{k}_{s}^{-} \cdot \mathbf{r}}, & z<-L / 2, \\
I_{\lambda}^{R} e^{i \mathbf{k}_{1}^{-} \cdot \mathbf{r}}+J_{\lambda}^{R} e^{i \mathbf{k}_{1}^{+} \cdot \mathbf{r}}, & |z|<L / 2, \\
e^{i \mathbf{k}^{-} \cdot \mathbf{r}}+R_{\lambda}^{R} e^{i \mathbf{k}^{+} \cdot \mathbf{r}}, & z>L / 2 .
\end{array}\right.
$$

For the sake of clarity the complete list of reflection and transmission coefficients is given in Appendix A. Here we only write the ones most relevant for the calculation at hand:

$$
\begin{aligned}
R_{\lambda}^{R} & =\frac{r_{\lambda}^{\mathrm{vl}}+r_{\lambda}^{\mathrm{ls}} e^{2 i k_{z 1} L}}{1+r_{\lambda}^{\mathrm{vl}} r_{\lambda}^{\mathrm{ls}} e^{2 i k_{z 1} L} e^{-i k_{z} L},} \\
T_{\lambda}^{L} & =\frac{t_{\lambda}^{\mathrm{sl}} t_{\lambda}^{\mathrm{lv}} e^{\left(2 i k_{z 1}-i k_{z \mathrm{~s}}-i k_{z}\right) L / 2}}{1+r_{\lambda}^{\mathrm{sl}} r_{\lambda}^{\mathrm{lv}} e^{2 i k_{z 1} L}} .
\end{aligned}
$$

\section{B. Trapped modes}

Trapped modes arise from repeated total internal reflections within the layer of higher refractive index $n_{1}$. This happens when the angle of incidence of the incoming wave is sufficiently high and exceeds the critical angle. This critical angle is different for the two opposite wave-guide interfaces. First consider the layer-vacuum interface. From Eq. (16) we can obtain the reciprocal relation expressing the $k_{z}$ in terms of the $k_{z 1}$ :

$$
k_{z}=\frac{1}{n_{1}} \sqrt{k_{z 1}^{2}-\left(n_{1}^{2}-1\right) \mathbf{k}_{\|}^{2}} .
$$

Thus, whenever $k_{z 1}^{2}<\left(n_{1}^{2}-1\right) \mathbf{k}_{\|}^{2}$ then $k_{z}$ becomes pure imaginary,

$$
k_{z}=+\frac{i}{n_{1}} \sqrt{\left(n_{1}^{2}-1\right) \mathbf{k}_{\|}^{2}-k_{z 1}^{2}},
$$

and we have a mode that exhibits evanescent behavior on the vacuum side. The sign of the square root is chosen such that these modes decay exponentially when one goes away from the layer in the positive $z$ direction. This also ensures that there truly is total internal reflection, that is, $\left|r_{\lambda}^{\mathrm{vl}}\right|^{2}=1$.

However, since on the other side of the wave guide we have a substrate rather than vacuum, not all of the modes that get totally internally reflected at the vacuum-layer interface necessarily get trapped. From the relation

$$
k_{z \mathrm{~s}}=\frac{n_{\mathrm{s}}}{n_{1}} \sqrt{k_{z 1}^{2}-\mathbf{k}_{\|}^{2}\left(\frac{n_{1}^{2}}{n_{\mathrm{s}}^{2}}-1\right)},
$$

we obtain the condition of total internal reflection for the substrate-layer interface to be $k_{z 1}^{2} \leqslant\left(n_{1}^{2} / n_{\mathrm{s}}^{2}-1\right) \mathbf{k}_{\|}^{2}$. Therefore, modes satisfying the condition

$$
\left(n_{1}^{2} / n_{\mathrm{s}}^{2}-1\right) \mathbf{k}_{\|}^{2} \leqslant k_{z 1}^{2} \leqslant\left(n_{1}^{2}-1\right) \mathbf{k}_{\|}^{2}
$$

are not trapped but appear in vacuum as a continuous spectrum of evanescent waves that are accounted for among the left-incident traveling modes. (They are analogous to the evanescent modes that occur at a single-interface half space, for which the normal-mode quantization was first presented in [7].) On the other hand, trapped modes occur if

$$
0 \leqslant k_{z 1}^{2} \leqslant\left(n_{1}^{2} / n_{\mathrm{s}}^{2}-1\right) \mathbf{k}_{\|}^{2} .
$$

The procedure for obtaining the trapped modes is largely equivalent to that of the traveling modes. They can be written in the form

$$
\mathbf{f}_{\mathbf{k} \lambda}^{T}(\mathbf{r})=N_{\lambda} \hat{\mathbf{e}}_{\lambda}(\nabla) \begin{cases}T_{\lambda}^{\mathrm{ls}} e^{i \mathbf{k}_{\mathrm{s}}^{-} \cdot \mathbf{r}}, & z<-L / 2, \\ V_{\lambda} e^{i \mathbf{k}_{1}^{-} \cdot \mathbf{r}}+e^{i \mathbf{k}_{1}^{+} \cdot \mathbf{r}}, & |z|<L / 2, \\ T_{\lambda}^{1 \mathrm{v}} e^{i \mathbf{k}^{+} \cdot \mathbf{r}}, & z>L / 2 .\end{cases}
$$

The boundary conditions are imposed on both interfaces. From the boundary at $z=-L / 2$ we get

$$
\begin{gathered}
T_{\lambda}^{\mathrm{ls}}=\left(t_{\lambda}^{\mathrm{ls}} / r_{\lambda}^{\mathrm{ls}}\right) e^{-i\left(k_{z 1}+k_{z \mathrm{~s}}\right) L / 2}, \\
V_{\lambda}=\left(1 / r_{\lambda}^{\mathrm{ls}}\right) e^{-i k_{z 1} L},
\end{gathered}
$$

whereas from the $z=L / 2$ boundary

$$
\begin{gathered}
T_{\lambda}^{\mathrm{lv}}=t_{\lambda}^{\mathrm{lv}} e^{-i\left(k_{z l}-k_{z}\right) L / 2}, \\
V_{\lambda}=r_{\lambda}^{\mathrm{lv}} e^{i k_{z 1} L} .
\end{gathered}
$$

Since both Eqs. (30) and (31) need to be simultaneously satisfied, we obtain a dispersion relation for these modes,

$$
1+r_{\lambda}^{\mathrm{vl}} r_{\lambda}^{\mathrm{ls}} e^{2 i k_{z 1} L}=0
$$

which determines the allowed values of $k_{z 1}$ within the layer. Since we are dealing with an atom on the vacuum side 
it is necessary to express the dispersion relation in terms of $k_{z}$ rather than $k_{z 1}$. It is straightforward to show that the allowed values of the $z$ component of the evanescent waves' wave vector appearing on the vacuum side are given by numbers $q_{\lambda}^{n}$ :

$$
\begin{gathered}
q_{\mathrm{TE}}^{n}=\left\{k_{z}: k_{z}+i k_{z 1}\left(k_{z}\right) \tan \left[\phi_{\mathrm{TE}}\left(k_{z}\right)\right]=0\right\}, \\
q_{\mathrm{TM}}^{n}=\left\{k_{z}: k_{z}+i k_{z 1}\left(k_{z}\right) / n_{1}^{2} \tan \left[\phi_{\mathrm{TM}}\left(k_{z}\right)\right]=0\right\},
\end{gathered}
$$

with

$$
\begin{gathered}
\phi_{\mathrm{TE}}\left(k_{z}\right)=\arg \left[\left(k_{z 1}+k_{z \mathrm{~s}}\right) e^{-i k_{z 1} L}\right], \\
\phi_{\mathrm{TE}}\left(k_{z}\right)=\arg \left[\left(k_{z 1} / n_{1}^{2}+k_{z \mathrm{~s}} / n_{\mathrm{s}}^{2}\right) e^{-i k_{z 1} L}\right] .
\end{gathered}
$$

The numbers $q_{\lambda}^{n}$ lie on the imaginary $k_{z}$ axis; they satisfy [cf. Eqs. (25) and (28)],

$$
\left(\frac{1}{n_{1}^{2}}-1\right) \mathbf{k}_{\|}^{2}<\left(q_{\lambda}^{n}\right)^{2}<\left(\frac{1}{n_{\mathrm{s}}^{2}}-1\right) \mathbf{k}_{\|}^{2} .
$$

The normalization constant $N_{\lambda}$ for trapped modes is easily obtained by direct evaluation of the integral (8). It is given by

$$
N_{\lambda}=\frac{1}{2 \pi}\left[2 n_{1}^{2} L+F_{\lambda}\left(n_{1}, n_{\mathrm{s}}\right)+F_{\lambda}\left(n_{1}, 1\right)\right]^{-1 / 2},
$$

with

$$
\begin{aligned}
F_{\lambda}\left(n_{1}, n_{\mathrm{s}}\right)= & \frac{n_{\mathrm{s}}^{2}}{2}\left|\hat{\mathbf{e}}_{\lambda}\left(\mathbf{k}_{\mathrm{s}}^{-}\right)\right|^{2} \frac{\left|t_{\lambda}^{\mathrm{ls}}\right|^{2}}{\left|k_{z \mathrm{~s}}\right|} \\
& -\frac{n_{1}}{k_{z 1}} \operatorname{Im}\left(\mathrm{r}_{\lambda}^{\mathrm{ls}}\right) \hat{\mathbf{e}}_{\lambda}^{*}\left(\mathbf{k}_{1}^{+}\right) \cdot \hat{\mathbf{e}}_{\lambda}\left(\mathbf{k}_{1}^{-}\right),
\end{aligned}
$$

and the reader is reminded that in (35) the $z$ components of the wave vectors $\mathbf{k}$ and $\mathbf{k}_{\mathrm{s}}$ are pure imaginary and because of that the TM polarization vectors $\hat{\mathbf{e}}_{\mathrm{TM}}\left(\mathbf{k}^{-}\right)$and $\hat{\mathbf{e}}_{\mathrm{TM}}\left(\mathbf{k}_{\mathrm{s}}^{-}\right)$are no longer normalized to unity, that is, $\left|\hat{\mathbf{e}}_{\mathrm{TM}}\left(\mathbf{k}_{\mathrm{s}}^{-}\right)\right|^{2} \neq 1$. The quantity $F_{\lambda}\left(n_{1}, 1\right)$ in Eq. (35) is obtained by taking the limit $n_{\mathrm{s}} \rightarrow 1$ of $F\left(n_{1}, n_{\mathrm{s}}\right)$, which, besides setting $n_{\mathrm{s}}=1$ explicitly, involves replacing $r_{\lambda}^{\mathrm{ls}}$ and $t_{\lambda}^{\mathrm{ls}}$ with $r_{\lambda}^{\mathrm{lv}}$ and $t_{\lambda}^{\mathrm{lv}}$ and $\mathbf{k}_{\mathrm{s}}$ with $\mathbf{k}$.

\section{Field operators and commutation relations: Completeness of the modes}

Now that we have determined the spatial dependence of the mode functions, we are in position to write out the vector potential field operator explicitly:

$$
\begin{aligned}
\hat{\mathbf{A}}(\mathbf{r}, t)= & \left\{\int d^{2} \mathbf{k}_{\|} \int_{0}^{\infty} d k_{z} \frac{1}{\sqrt{2 \epsilon_{0} \omega_{\mathbf{k}}}} \mathbf{f}_{\mathbf{k} \lambda}^{R}(\mathbf{r}) a_{\mathbf{k} \lambda}^{R} e^{-i \omega_{\mathbf{k}} t}\right. \\
& +\int d^{2} \mathbf{k}_{\|} \int_{0}^{\infty} d k_{z \mathrm{~s}} \frac{1}{\sqrt{2 \epsilon_{0} \omega_{\mathbf{k}}}} \mathbf{f}_{\mathbf{k} \lambda}^{L}(\mathbf{r}) a_{\mathbf{k} \lambda}^{L} e^{-i \omega_{\mathbf{k}} t} \\
& \left.+\int d^{2} \mathbf{k}_{\|} \sum_{k_{z 1}} \frac{1}{\sqrt{2 \epsilon_{0} \omega_{\mathbf{k}}}} \mathbf{f}_{\mathbf{k} \lambda}^{T}(\mathbf{r}) a_{\mathbf{k} \lambda}^{T} e^{-i \omega_{\mathbf{k}} t}\right\}+ \text { H.c. }
\end{aligned}
$$

The sum in the last term runs over the allowed values of the $z$ component of the layer's wave vector $k_{z 1}$, that is, the solutions of the dispersion relation (32). For a given type of mode, left-incident, right-incident, or trapped, photon creation and annihilation operators appearing in (36) satisfy the commutation relations (10). Commutators between photon operators corresponding to different types of modes vanish as a consequence of the orthogonality of the field modes (8), for example,

$$
\left[a_{\mathbf{k} \lambda}^{L},\left(a_{\mathbf{k}^{\prime} \lambda^{\prime}}^{R}\right)^{\dagger}\right]=0 .
$$

We would like to verify explicitly the equal-time canonical commutation relation between field operators, say, between the electric field operator $\hat{\mathbf{E}}(\mathbf{r}, t)$ and the vector potential operator $\hat{\mathbf{A}}(\mathbf{r}, t)$,

$$
\left[\hat{A}_{i}(\mathbf{r}, t), \epsilon_{0} \hat{E}_{j}\left(\mathbf{r}^{\prime}, t\right)\right]=-i \delta_{i j}^{\epsilon}\left(\mathbf{r}, \mathbf{r}^{\prime}\right), \quad z, z^{\prime}>L / 2,
$$

with $\delta_{i j}^{\epsilon}\left(\mathbf{r}, \mathbf{r}^{\prime}\right)$ given by Eqs. (6) and (7). To evaluate (38) we need the electric field operator which is easily obtained from Eq. (36) using the relation $\mathbf{E}=-\partial_{t} \mathbf{A}$. Plugging in the field operators into (38) and making use of commutation relations (10) and (37), we find that the left-hand side (LHS) of (38) is given by

$$
\begin{aligned}
\mathrm{LHS}= & i \operatorname{Re} \sum_{\lambda} \int d^{2} \mathbf{k}_{\|}\left[\int_{0}^{\infty} d k_{z} f_{\mathbf{k} \lambda, i}^{R}(\mathbf{r}) f_{\mathbf{k} \lambda, j}^{* R}\left(\mathbf{r}^{\prime}\right)\right. \\
& +\int_{0}^{\infty} d k_{z \mathrm{~s}} f_{\mathbf{k} \lambda, i}^{L}(\mathbf{r}) f_{\mathbf{k} \lambda, j}^{* L}\left(\mathbf{r}^{\prime}\right) \\
& \left.+\sum_{k_{z 1}} f_{\mathbf{k} \lambda, i}^{T}(\mathbf{r}) f_{\mathbf{k} \lambda, j}^{* T}\left(\mathbf{r}^{\prime}\right)\right]
\end{aligned}
$$

The quantity on the right-hand side is the sum over all modes, just as prescribed by Eq. (5), and therefore we expect it to be equal to the generalized transverse $\delta$ function [Eq. (6)]. This shows that the statement of the completeness of the modes (5) is in fact equivalent to the commutation relation (38), as has been noted before in [18]. To prove that the relation

$$
\begin{aligned}
\delta_{i j}^{\epsilon}\left(\mathbf{r}, \mathbf{r}^{\prime}\right)= & \sum_{\lambda} \int d^{2} \mathbf{k}_{\|}\left[\int_{0}^{\infty} d k_{z} f_{\mathbf{k} \lambda, i}^{R}(\mathbf{r}) f_{\mathbf{k} \lambda, j}^{* R}\left(\mathbf{r}^{\prime}\right)\right. \\
& +\int_{0}^{\infty} d k_{z \mathrm{~s}} f_{\mathbf{k} \lambda, i}^{L}(\mathbf{r}) f_{\mathbf{k} \lambda, j}^{* L}\left(\mathbf{r}^{\prime}\right) \\
& \left.+\sum_{k_{z 1}} f_{\mathbf{k} \lambda, i}^{T}(\mathbf{r}) f_{\mathbf{k} \lambda, j}^{* T}\left(\mathbf{r}^{\prime}\right)\right]
\end{aligned}
$$

holds for $z, z^{\prime}>L / 2$ we need to work out the sum over all field modes. To start with, we carry out a change of variables in (40): We convert the $k_{z \mathrm{~s}}$ integral and the $k_{z 1}$ sum to run over the values of $k_{z}$. In the case of the $k_{z \mathrm{~s}}$ integral this is a simple change of variables according to (17)

$$
\int_{0}^{\infty} d k_{z \mathrm{~s}}=n_{\mathrm{s}}^{2} \int_{0}^{\infty} d k_{z} \frac{k_{z}}{k_{z \mathrm{~s}}}+n_{\mathrm{s}}^{2} \int_{i \Gamma_{\mathrm{s}}}^{0} d k_{z} \frac{k_{z}}{k_{z \mathrm{~s}}},
$$

with $\Gamma_{\mathrm{s}}=\sqrt{\left(n_{\mathrm{s}}^{2}-1\right) \mathbf{k}_{\|}^{2}} / n_{\mathrm{s}}$. Here it is seen explicitly that the contributions from the left-incident modes split into a traveling part and an evanescent part. The values of $k_{z}$ included in the last integral correspond to the condition for evanescent modes with continuous spectrum [Eq. (27)]. In the case of the sum we change the summation over $k_{z 1}$ to run over the values of $k_{z}$ as defined by Eq. (33). Plugging in the mode functions (20) and (21) into Eq. (40) and utilizing straightforward properties of 
the reflection and transmission coefficients that hold for real $k_{z}, k_{z \mathrm{~s}}$,

$$
R_{\lambda}^{* R}\left(-k_{z}\right)=R_{\lambda}^{R}\left(k_{z}\right), \quad \frac{k_{z}}{k_{z \mathrm{~s}}}\left|T_{\lambda}^{L}\right|^{2}+\left|R_{\lambda}^{R}\right|^{2}=1,
$$

we can rewrite the completeness relation as

$$
\begin{aligned}
\delta_{i j}^{\epsilon}\left(\mathbf{r}, \mathbf{r}^{\prime}\right)= & \delta_{i j}^{\perp}\left(\mathbf{r}-\mathbf{r}^{\prime}\right)+\sum_{\lambda} \hat{e}_{\lambda}^{i}(\nabla) \hat{e}_{\lambda}^{* j}\left(\nabla^{\prime}\right) \int d^{2} \mathbf{k}_{\|} e^{i \mathbf{k}_{\|}\left(\mathbf{r}_{\|}-\mathbf{r}_{\|}^{\prime}\right)} \\
& \times\left\{\sum_{q_{\lambda}^{n}}\left|N_{\lambda}\right|^{2}\left|T_{\lambda}^{\mathrm{lv}}\right|^{2} e^{i k_{z}\left(z+z^{\prime}\right)}\right. \\
& +\frac{1}{(2 \pi)^{3}} \int_{i \Gamma_{\mathrm{s}}}^{0} d k_{z} \frac{k_{z}}{k_{z \mathrm{~s}}}\left|T_{\lambda}^{L}\right|^{2} e^{i k_{z}\left(z+z^{\prime}\right)} \\
& \left.+\frac{1}{(2 \pi)^{3}} \int_{-\infty}^{\infty} d k_{z} R_{\lambda}^{R} e^{i k_{z}\left(z+z^{\prime}\right)}\right\}
\end{aligned}
$$

The first term in the preceding equation is the standard transverse $\delta$ function. Therefore, if Eq. (40) is to hold, the term in the curly brackets needs to be proportional to the reflection part of the electrostatic Green's function [cf. the second term on the RHS of Eq. (7)]. That this is indeed the case is at this stage far from obvious, as for the proof one would need to combine two integrals and a sum into one expression. Obviously, the discreteness of the spectrum of the trapped modes is a nuisance that needs to be overcome if one is to complete the task of summing over the electromagnetic modes successfully. A similar difficulty would arise in any perturbative calculation in this type of geometry, which motivated a previous investigation of this problem for the symmetric case of a single slab of dielectric material [17]. We proceed with a broadly analogous method to [17], first noting that what we have here can be considered as a superposition of a slab and a half-space geometry (cf. [17] and [18]). One can utilize the branch cut due to $k_{z s}$ [which runs along the imaginary $k_{z}$ axis between $\pm i \Gamma_{\mathrm{s}}$ (cf. Fig. 2)] to express the integral over $\left|T_{\lambda}^{L}\right|^{2}$ in (43) as an integral over the reflection coefficient $R_{\lambda}^{R}$ that runs from $0^{-}$ along the square root cut up to the branch point at $+i \Gamma_{\mathrm{s}}$ and then back down to the origin $0^{+}$. Note that the branch cut due to the $k_{z 1}$ is irrelevant because of the symmetry property of the reflection coefficient $R_{\lambda}^{R}\left(-k_{z 1}\right)=R_{\lambda}^{R}\left(k_{z 1}\right)$. In this way, the first two integrals in the curly braces in Eq. (43) can be combined together as a single integral in the complex $k_{z}$ plane [18]. This is possible because the relation

$$
\left.\frac{k_{z}}{k_{z \mathrm{~s}}}\left|T_{\lambda}^{L}\right|^{2}\right|_{k_{z \mathrm{~s}}, k_{z 1}>0}=\left.R_{\lambda}^{R}\right|_{k_{z \mathrm{~s}}, k_{z 1}>0}-\left.R_{\lambda}^{R}\right|_{k_{z \mathrm{~s}}, k_{z 1}<0}
$$

continues to hold for coefficients (22) with a purely imaginary $z$ component of the vacuum wave vector, $k_{\mathrm{z}}$ (cf. [19]). Thus, the contributions from the traveling and evanescent modes can be combined into a single contour integral along the path $\gamma_{\mathrm{s}}$ depicted in Fig. 2 and the terms appearing in the curly brackets on the RHS of Eq. (43) become

$$
\begin{aligned}
& \frac{1}{(2 \pi)^{3}} \int_{\gamma_{\mathrm{s}}} d k_{z} R_{\lambda}^{R} \hat{e}_{\lambda}^{i}\left(\mathbf{k}^{+}\right) \hat{e}_{\lambda}^{j}\left(\mathbf{k}^{-}\right) e^{i k_{z}\left(z+z^{\prime}\right)} \\
& \quad+\sum_{q_{\lambda}^{n}}\left|N_{\lambda}\right|^{2}\left|T_{\lambda}^{\mathrm{lv}}\right|^{2} \hat{e}_{\lambda}^{i}\left(\mathbf{k}^{+}\right) \hat{e}_{\lambda}^{j}\left(\mathbf{k}^{-}\right) e^{i k_{z}\left(z+z^{\prime}\right)} .
\end{aligned}
$$

Here we have now included the polarization vectors explicitly in the integrals, which is a crucial step as they affect the

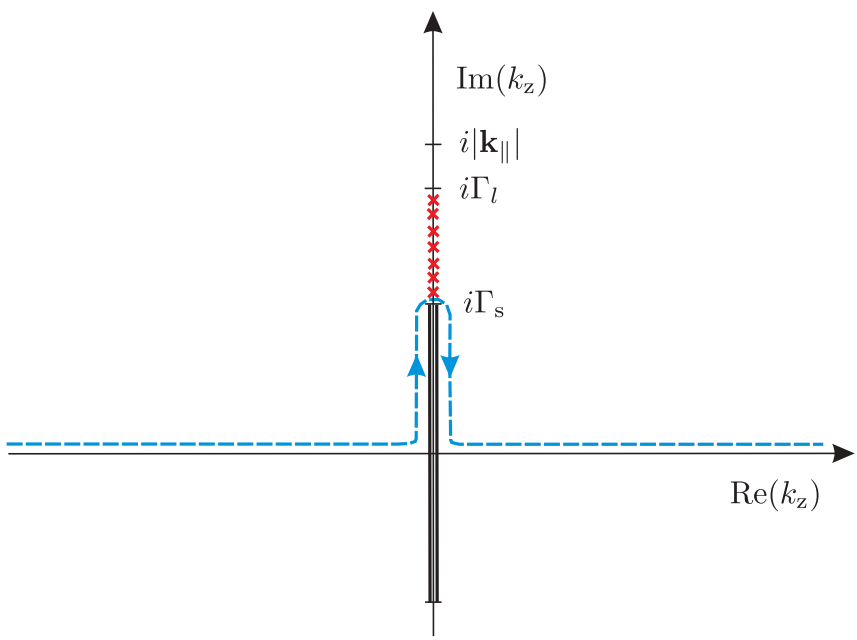

FIG. 2. (Color online) The dashed line represents the contour $\gamma_{\mathrm{s}}$ used to evaluate the $k_{z}$ integral in Eq. (45). Here $\Gamma_{\mathrm{s}}=\sqrt{\left(n_{\mathrm{s}}^{2}-1\right) \mathbf{k}_{\|}^{2}} / n_{\mathrm{s}}$ and $\Gamma_{1}=\sqrt{\left(n_{1}^{2}-1\right) \mathbf{k}_{\|}^{2}} / n_{1}$. The crosses represent the poles of the reflection coefficient $R_{\lambda}^{R}$, that is, the solutions to the dispersion relation (32).

analytical structure of the integrand in the complex $k_{z}$ plane. In particular, the TM polarization vector introduces a pole at the points $k_{\mathrm{z}}= \pm i\left|\mathbf{k}_{\|}\right|$due to the factor $1 /|\mathbf{k}|^{2}$ in its normalization factor. We will see that it is precisely this pole that gives rise to the reflection term in (6). We note that, according to Eq. (22), the reflection coefficient contains the phase factor $e^{-i k_{z} L}$. Thus, since $z+z^{\prime}-L>0$, the argument of the exponential in (45) has a negative real part in the upper half of the complex $k_{z}$ plane and we can evaluate the $k_{z}$ integral in Eq. (45) by closing the contour in the upper half plane. For this we need to determine the analytical properties of $R_{\lambda}^{R}$. We note that the denominator of the reflection coefficient (22) is precisely the dispersion relation (32). Rewriting the reflection coefficients in the form

$$
\begin{gathered}
R_{\mathrm{TE}}^{R}=\frac{k_{z}-k_{z 1}\left(\frac{1-r_{\mathrm{T}}^{\mathrm{ls}} \exp \left(2 i k_{z 1} L\right)}{1+r_{\mathrm{TI}}^{\mathrm{l}} \exp \left(2 i k_{z 1} L\right)}\right)}{k_{z}+k_{z 1}\left(\frac{1-r_{\mathrm{T}}^{\mathrm{s}} \exp \left(2 i k_{z 1} L\right)}{1+r_{\mathrm{TE}}^{\mathrm{l}} \exp \left(2 i k_{z 1} L\right)}\right)}, \\
R_{\mathrm{TM}}^{R}=\frac{k_{z}-\frac{k_{z 1}}{n_{1}^{2}}\left(\frac{1-r_{\mathrm{TM}}^{\mathrm{ls}} \exp \left(2 i k_{z 1} L\right)}{1+r_{\mathrm{TM}}^{\mathrm{s}} \exp \left(2 i k_{z 1} L\right)}\right)}{k_{z}+\frac{k_{z 1}}{n_{1}^{2}}\left(\frac{1-r_{\mathrm{TM}}^{\mathrm{ls}} \exp \left(2 i k_{z 1} L\right)}{1+r_{\mathrm{TM}}^{\mathrm{Is}} \exp \left(2 i k_{z 1} L\right)}\right)}
\end{gathered}
$$

allows us to deduce that $R_{\lambda}^{R}$ has a finite number of simple poles on the imaginary axis. When closing the contour we enclose all of them and by Cauchy's theorem the problem is reduced to the evaluation of the residues at these points:

$$
\begin{aligned}
& \sum_{\lambda} \int_{\gamma_{\mathrm{s}}} d k_{z} R_{\lambda}^{R} \hat{e}_{\lambda}^{i}\left(\mathbf{k}^{+}\right) \hat{e}_{\lambda}^{j}\left(\mathbf{k}^{-}\right) e^{i k_{z}\left(z+z^{\prime}\right)} \\
& =2 \pi i \sum_{\lambda} \sum_{\operatorname{Res}} R_{\lambda}^{R} \hat{e}_{\lambda}^{i}\left(\mathbf{k}^{+}\right) \hat{e}_{\lambda}^{j}\left(\mathbf{k}^{-}\right) e^{i k_{z}\left(z+z^{\prime}\right)} \\
& =2 \pi i\left[\sum_{\lambda} \sum_{q_{\lambda}^{n}} \lim _{k_{z} \rightarrow q_{\lambda}^{n}}\left(k_{z}-q_{\lambda}^{n}\right)+\lim _{k_{z} \rightarrow i\left|\mathbf{k}_{\|}\right|}\left(k_{z}-i\left|\mathbf{k}_{\|}\right|\right)\right] \\
& \quad \times \hat{e}_{\lambda}^{i}\left(\mathbf{k}^{+}\right) \hat{e}_{\lambda}^{j}\left(\mathbf{k}^{-}\right) \frac{r_{\lambda}^{\mathrm{vl}}+r_{\lambda}^{\mathrm{ls}} e^{2 i k_{\mathrm{zl}} L}}{1+r_{\lambda}^{\mathrm{vl}} r_{\lambda}^{\mathrm{ls}} e^{2 i k_{\mathrm{zl}} L}} e^{i k_{z}\left(z+z^{\prime}-L\right)} .
\end{aligned}
$$


Here the first term represents the contributions from the poles in the reflection coefficient and corresponds the trapped modes, whereas the second term represents the contribution from the pole that arises due to the TM polarization vector. When calculating the residues explicitly, one needs to remember that the two independent variables are $k_{z}$ and $\mathbf{k}_{\|}$and that, according to Eqs. (16) and (17), $k_{z 1}$ and $k_{z \mathrm{~s}}$ are functions of those. In addition, the denominator of the reflection coefficient is not of the form $f\left(k_{z}\right)\left(k_{z}-q_{\lambda}^{n}\right)$, so that multiplying it by $\left(k_{z}-q_{\lambda}^{n}\right)$ does not remove its singularity; the whole expression is still indeterminate. Therefore, L'Hospital's rule needs to be used to evaluate the limit (cf. [17], Sec. V]). Doing so, we find that

$$
\begin{aligned}
& \frac{1}{(2 \pi)^{3}} \int_{\gamma_{\mathrm{s}}} d k_{z} R_{\lambda}^{R} \hat{e}_{\lambda}^{i}\left(\mathbf{k}^{+}\right) \hat{e}_{\lambda}^{j}\left(\mathbf{k}^{-}\right) e^{i k_{z}\left(z+z^{\prime}\right)} \\
& =-\sum_{q_{\lambda}^{n}}\left|N_{\lambda}\right|^{2}\left|T_{\lambda}^{\mathrm{lv}}\right|^{2} \hat{e}_{\lambda}^{i}\left(\mathbf{k}^{+}\right) \hat{e}_{\lambda}^{j}\left(\mathbf{k}^{-}\right) e^{i k_{z}\left(z+z^{\prime}\right)} \\
& \quad-\nabla_{i} \nabla_{j}^{\prime} G_{H}\left(\mathbf{r}, \mathbf{r}^{\prime}\right),
\end{aligned}
$$

where $G_{H}\left(\mathbf{r}, \mathbf{r}^{\prime}\right)$ is the reflected part of the Green's function of the Poisson equation given in Eq. (7) and derived in Appendix B. We see that the poles of the reflection coefficient $R_{\lambda}^{R}$ yield a term that exactly cancels out the contributions of the trapped modes to the completeness relation (43), whereas the pole of the TM polarization vector yields the term proportional to Green's function. Thus, the final result can be written as

$$
\begin{gathered}
\sum_{\lambda} \int d^{2} \mathbf{k}_{\|} \int_{k_{z}} f_{\mathbf{k} \lambda}^{i}(\mathbf{r}) f_{\mathbf{k} \lambda}^{* j}\left(\mathbf{r}^{\prime}\right)=\frac{1}{i}\left[A_{i}(\mathbf{r}),-\epsilon_{0} E_{j}\left(\mathbf{r}^{\prime}\right)\right] \\
=\delta_{i j}^{\perp}\left(\mathbf{r}-\mathbf{r}^{\prime}\right)-\nabla_{i} \nabla_{j}^{\prime} G_{H}\left(\mathbf{r}, \mathbf{r}^{\prime}\right) \quad z, z^{\prime}>L / 2 \\
=\delta_{i j} \delta^{(3)}\left(\mathbf{r}-\mathbf{r}^{\prime}\right)-\nabla_{i} \nabla_{j}^{\prime} G\left(\mathbf{r}, \mathbf{r}^{\prime}\right) \quad z, z^{\prime}>L / 2,
\end{gathered}
$$

which is precisely what we anticipated earlier. In the next section we demonstrate how the calculation presented here may be applied to accomplish typical perturbative QED calculations in a layered geometry.

\section{ENERGY SHIFT}

To work out the energy shift we use standard perturbation theory where the atom is treated by means of the Schrödinger quantum mechanics and only the electromagnetic field is second quantized. We work with a multipolar coupling where the lowest order of the interaction Hamiltonian is

$$
H_{\text {int }}=-\boldsymbol{\mu} \cdot \mathbf{E} \text {. }
$$

Then the energy shift of the atomic state $i$, up to the second order, is given by

$$
\Delta E_{i}=\left\langle i ; 0\left|H_{\mathrm{int}}\right| i ; 0\right\rangle+\sum_{j \neq i} \sum_{\mathbf{k}, \lambda} \frac{\left|\left\langle j ; 1_{\mathbf{k} \lambda}\left|H_{\mathrm{int}}\right| i ; 0\right\rangle\right|^{2}}{E_{i}-\left(E_{j}+\omega_{\mathbf{k}}\right)} .
$$

Here, $\boldsymbol{\mu}$ is the atomic electric dipole moment, and the composite state $\left|j ; 1_{\mathbf{k} \lambda}\right\rangle$ describes the atom in the state $|j\rangle$ with energy $E_{j}$ and the photon field containing one photon with momentum $\mathbf{k}$ and polarization $\lambda$. Because the electric field operator is linear in the photon creation and annihilation operators, the first-order contribution vanishes and the secondorder correction is the lowest-order contribution. Since the electric field does not vary appreciably over the size of the atom, we use the electric dipole approximation. Then the energy shift can be expressed as

$$
\Delta E_{i}=-\sum_{j \neq i} \sum_{\mathbf{k}, \lambda} \frac{\omega_{\mathbf{k}}}{2 \epsilon_{0}} \frac{\left|\langle i|\boldsymbol{\mu}| j\rangle \cdot \mathbf{f}_{\mathbf{k} \lambda}^{*}\left(\mathbf{r}_{0}\right)\right|^{2}}{E_{j i}+\omega_{\mathbf{k}}}
$$

where $\mathbf{r}_{0}=\left(0,0, z_{0}\right)$ is the position of the atom and we have abbreviated $E_{j i}=E_{j}-E_{i}$. It is seen that the calculation involves a summation over the modes of the electromagnetic field as carried out in the proof of the completeness relation (43). Equation (49) can be written out explicitly as

$$
\begin{aligned}
\Delta E_{i}= & -\frac{1}{2 \epsilon_{0}} \sum_{\lambda} \sum_{j \neq i}\left|\mu_{m}\right|^{2} \int d \mathbf{k}_{\|} \\
& \times\left(\Delta^{\mathrm{vac}}+\Delta^{\text {trav }}+\Delta^{\text {evan }}+\Delta^{\text {trap }}\right),
\end{aligned}
$$

with $\left|\mu_{m}\right|^{2} \equiv\left|\left\langle i\left|\mu_{m}\right| j\right\rangle\right|^{2}$. There are four distinct contributions to the energy shift. $\Delta^{\mathrm{vac}}$ is the position-independent contribution caused by the vacuum fields and gives rise to the Lamb shift in free space:

$$
\Delta^{\mathrm{vac}}=\frac{1}{(2 \pi)^{3}} \int_{-\infty}^{\infty} d k_{z} e_{\lambda}^{m}\left(\mathbf{k}^{-}\right) e_{\lambda}^{m *}\left(\mathbf{k}^{-}\right) \frac{\omega}{E_{j i}+\omega} .
$$

The remaining three contributions come from the traveling, evanescent, and trapped modes, respectively,

$$
\begin{aligned}
\Delta^{\mathrm{trav}}= & \frac{1}{(2 \pi)^{3}} \int_{-\infty}^{\infty} d k_{z} R_{\lambda}^{R} e_{\lambda}^{m}\left(\mathbf{k}^{+}\right) e_{\lambda}^{m *}\left(\mathbf{k}^{-}\right) e^{2 i k_{z} z_{0}} \frac{\omega}{E_{j i}+\omega}, \\
\Delta^{\mathrm{evan}}= & \frac{1}{(2 \pi)^{3}} \int_{i \Gamma_{\mathrm{s}}}^{0} d k_{z} \frac{k_{z}}{k_{z \mathrm{~s}}}\left|T_{\lambda}^{L}\right|^{2} e_{\lambda}^{m}\left(\mathbf{k}^{+}\right) e_{\lambda}^{m *}\left(\mathbf{k}^{+}\right) e^{2 i k_{z} z_{0}} \\
& \times \frac{\omega}{E_{j i}+\omega}, \\
\Delta^{\mathrm{trap}}= & -2 m m \sum_{q_{\lambda}^{n}}\left|N_{\lambda}\right|^{2}\left|T_{\lambda}^{\mathrm{lv}}\right|^{2} e_{\lambda}^{m}\left(\mathbf{k}^{+}\right) e_{\lambda}^{m *}\left(\mathbf{k}^{+}\right) e^{2 i k_{z} z_{0}} \frac{\omega}{E_{j i}+\omega},
\end{aligned}
$$

with $z_{0}$ being the position of the atom with respect to the origin. Note that because of the dipole approximation the shorthand notation for polarization vectors (19) can no longer be applied. Normally one is interested in the energy shift caused by the presence of the dielectric boundaries only; that is, the correction to the shift that would appear in the free space. Therefore, we renormalize the energy-level shift (50) by subtracting from it its free space limit, that is,

$$
\Delta E_{i}^{\mathrm{ren}}=\Delta E_{i}-\lim _{n_{1}, n_{\mathrm{s}} \rightarrow 1} \Delta E_{i} .
$$

The renormalization procedure amounts to the removal of the contributions $\Delta^{\mathrm{vac}}$ [Eq. (51)], from the energy shift (50) and takes care of any infinities that would appear otherwise, provided we treat the remaining parts with care. As noted elsewhere [1], the contributions (52) suffer from convergence problems when treated separately. However, appropriate tools for handling the problem have been developed in Sec. II C. We aim to combine $\Delta^{\text {trav }}, \Delta^{\text {evan }}$, and $\Delta^{\text {trap }}$ into one compact expression that is easy to handle analytically. We can use the same trick as in the proof of the completeness relation because the analytical structure of the integrand in the complex $k_{z}$ plane is the same except for the function $\omega=\left(\mathbf{k}_{\|}^{2}+k_{z}^{2}\right)^{1 / 2}$ that comes about due to the denominator of perturbation theory and introduces additional branch points at $k_{z}= \pm i\left|\mathbf{k}_{\|}\right|$ as compared to Fig. 2. This poses no difficulties, though, if 


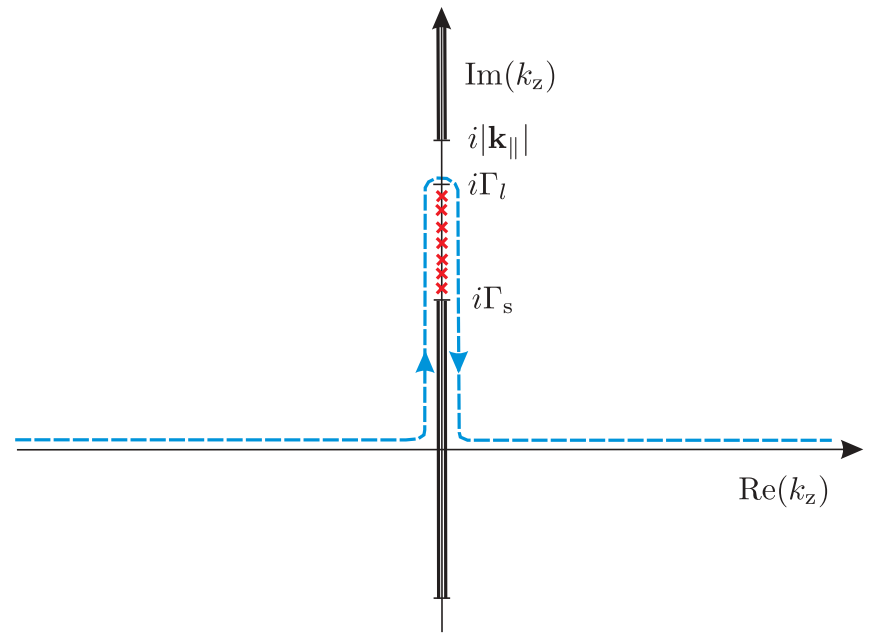

FIG. 3. (Color online) The dashed line represents the final contour $\gamma_{1}$ used to evaluate the energy shift in Eq. (54).

one chooses the branch cuts to lie between $\pm i\left|\mathbf{k}_{\|}\right|$and $\pm i \infty$. Then, the contributions to the energy shift from the traveling modes $\Delta^{\text {trav }}$ and the evanescent modes $\Delta^{\text {evan }}$ can be combined together into a single complex integral, as explained in the steps between Eqs. (43) and (45). This is possible because for imaginary $k_{z}$ we have $e_{\lambda}^{m *}\left(\mathbf{k}^{+}\right)=e_{\lambda}^{m}\left(\mathbf{k}^{-}\right)$, whereas for real $k_{z}$ the relation $e_{\lambda}^{m *}\left(\mathbf{k}^{-}\right)=e_{\lambda}^{m}\left(\mathbf{k}^{-}\right)$holds. On the other hand, we also know from Eq. (47) that the sum in $\Delta^{\text {trap }}$ is equal to an integral over the reflection coefficient $R_{\lambda}^{R}$ taken along any clockwise contour enclosing all of its poles. Choosing this contour to run from $k_{z}=0^{-}+i \Gamma_{\mathrm{s}}$ to $k_{z}=0^{-}+i \Gamma_{1}$ and then back down from $k_{z}=0^{+}+i \Gamma_{1}$ to $k_{z}=0^{+}+i \Gamma_{\mathrm{s}}$ (cf. Fig. 3), we write the renormalized energy shift compactly as

$$
\begin{aligned}
\Delta E_{i}^{\mathrm{ren}}= & -\frac{1}{2(2 \pi)^{3} \epsilon_{0}} \sum_{m, \lambda} \sum_{j \neq i}\left|\mu_{m}\right|^{2} \int d \mathbf{k}_{\|} \\
& \times \int_{\gamma_{1}} d k_{z} \frac{\omega}{E_{j i}+\omega} R_{\lambda}^{R} e_{\lambda}^{m}\left(\mathbf{k}^{+}\right) e_{\lambda}^{m}\left(\mathbf{k}^{-}\right) e^{2 i k_{z} z_{0}},
\end{aligned}
$$

where the contour of integration $\gamma_{1}$ is shown in Fig. 3. It resembles that of Fig. 2 but now runs on the imaginary axis up to the point $k_{z}=i \Gamma_{1}$ enclosing all the poles of the reflection coefficients $R_{\lambda}^{R}$. Formula (54) is equally applicable to ground-state atoms $|0\rangle$ as it is to atoms that are in an excited state $|i\rangle$ provided we use the contour of integration as given in Fig. 3 and interpret the $k_{z}$ integral as a Cauchy principal value. As renormalization has now been dealt with, we from here on omit the superscript "ren" and designate the renormalized energy shift of Eq. (54) simply by $\Delta E_{i}$.

\section{A. Ground-state atoms}

In the case of a ground-state atom the energy difference $E_{j 0} \equiv E_{j}-E_{0}$ is always positive; hence, the denominator in Eq. (54) that originates from second-order perturbation theory, $E_{j 0}+\omega$, never vanishes. Then the integrand in Eq. (54) contains no poles in the upper half of the $k_{z}$ plane other than those due to the reflection coefficient $R_{\lambda}^{R}$. To evaluate the $k_{z}$ integral we can deform the contour of integration in Eq. (54) from that sketched in Fig. 3 to the one as shown in Fig. 4, which is beneficial from the computational point

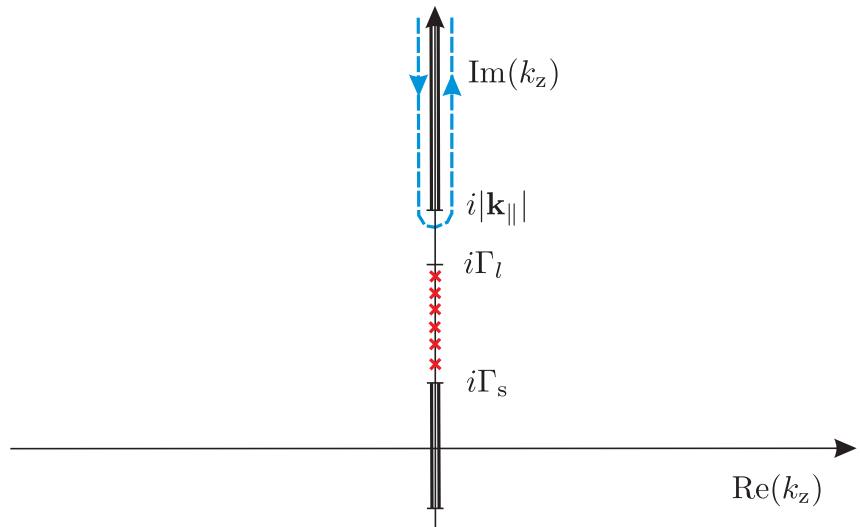

FIG. 4. (Color online) The final contour $\mathcal{C}$ used to evaluate the energy shift of the ground-state atom in Eq. (55).

of view as it simplifies the analysis of Eq. (54) considerably. Writing out explicitly the sums over the polarization vectors (19) and then expressing the integral in the $\mathbf{k}_{\|}$plane in polar coordinates, $k_{x}=k_{\|} \cos \phi, k_{y}=k_{\|} \sin \phi$, where the angle integral is computable analytically, we rewrite the energy shift as

$$
\begin{aligned}
\Delta E_{0}= & \frac{1}{16 \pi^{2} \epsilon_{0}} \sum_{j \neq 0} \int_{0}^{\infty} d k_{\|} k_{\|} \int_{\mathcal{C}} d k_{z} \frac{\omega}{E_{j 0}+\omega} e^{2 i k_{z} \mathcal{Z}} \\
& \times\left[\left|\mu_{\|}\right|^{2}\left(\tilde{R}_{\mathrm{TE}}^{R}-\frac{k_{z}^{2}}{\omega^{2}} \tilde{R}_{\mathrm{TM}}^{R}\right)+2\left|\mu_{\perp}\right|^{2} \frac{k_{\|}^{2}}{\omega^{2}} \tilde{R}_{\mathrm{TM}}^{R}\right],
\end{aligned}
$$

with $\omega\left(k_{z}\right)=\sqrt{k_{\|}^{2}+k_{z}^{2}}$ and $\left|\mu_{\|}\right|^{2}=\left|\mu_{x}\right|^{2}+\left|\mu_{y}\right|^{2}$ and the contour $\mathcal{C}$ is that in Fig. 4. The amended reflection coefficients $\tilde{R}_{\lambda}^{R}$ are given by

$$
\tilde{R}_{\lambda}^{R}=\frac{r_{\lambda}^{\mathrm{vl}}+r_{\lambda}^{\mathrm{ls}} e^{2 i k_{\mathrm{zl}} L}}{1+r_{\lambda}^{\mathrm{vl}} r_{\lambda}^{\mathrm{ls}} e^{2 i k_{\mathrm{zl}} L}}
$$

that is, we have pulled out the phase factor $e^{-i k_{z} L}$ in order to define $\mathcal{Z}=z_{0}-L / 2$ as the distance between the atom and the surface [cf. Eq. (22)].

In order to perform the $k_{z}$ integration in (55) we need to analytically continue the function $\omega=\omega\left(k_{z}\right)$, which is real and positive on the real axis, to the both sides of the branch cut along which the integration is carried out (cf. Fig. 4). Doing so we find that on the LHS of the cut the positive value of the square root needs to be taken, and hence on the RHS of the cut we must take the opposite sign. Therefore, we have

$$
\int_{C} d k_{z} \frac{\omega}{E_{j 0}+\omega}=-\int_{i k_{\|}}^{i \infty} d k_{z} \frac{2 E_{j 0} \omega}{\left(E_{j 0}-\omega\right)\left(E_{j 0}+\omega\right)} .
$$

Now we carry out a sequence of changes of variables. First we re-express the $k_{z}$ integration in terms of one over the frequency $\omega$ by substituting $\omega=\sqrt{k_{\|}^{2}+k_{z}^{2}}$,

$$
\int_{i k_{\|}}^{i \infty} d k_{z}=\int_{0}^{i \infty} d \omega \frac{\omega}{\sqrt{\omega^{2}-k_{\|}^{2}}} .
$$

Then we make the integral run along the real axis by setting $\omega=i \xi$. After this is done, the energy shift of the ground state 
is expressed as a double integral that covers the first quadrant of the $\left(k_{\|}, \xi\right)$ plane:

$$
\begin{aligned}
\Delta E_{0}= & -\frac{1}{8 \pi^{2} \epsilon_{0}} \sum_{j \neq 0} E_{j 0} \int_{0}^{\infty} d k_{\|} k_{\|} \\
& \times \int_{0}^{\infty} d \xi \frac{e^{-2 \sqrt{\xi^{2}+k_{\|}^{2}} \mathcal{Z}}}{\sqrt{\xi^{2}+k_{\|}^{2}}\left(E_{j 0}^{2}+\xi^{2}\right)} \\
& \times\left\{\left|\mu_{\|}\right|^{2}\left[\left(\xi^{2}+k_{\|}^{2}\right) \tilde{R}_{\mathrm{TM}}^{R}-\xi^{2} \tilde{R}_{\mathrm{TE}}^{R}\right]+2 k_{\|}^{2} \tilde{R}_{\mathrm{TM}}^{R}\left|\mu_{\perp}\right|^{2}\right\} .
\end{aligned}
$$

It seems natural to introduce polar coordinates, $k_{\|}=$ $\bar{x} \sin \phi, \xi=\bar{x} \cos \phi$. We also choose to scale the radial integration variable $\bar{x}=E_{j 0} x$ with $E_{j 0}>0$ and set $y=\cos \phi$. This provides us with the final form of the energy shift that is more suitable for numerical computations and asymptotic analysis:

$$
\begin{aligned}
\Delta E_{0}= & \frac{1}{8 \pi^{2} \epsilon_{0}} \sum_{j \neq 0} E_{j 0}^{3} \int_{0}^{\infty} d x x^{3} \int_{0}^{1} d y \frac{e^{-2 E_{j 0} \mathcal{Z} x}}{1+x^{2} y^{2}} \\
& \times\left[\left|\mu_{\|}\right|^{2}\left(y^{2} \tilde{R}_{\mathrm{TE}}^{R}-\tilde{R}_{\mathrm{TM}}^{R}\right)+2\left|\mu_{\perp}\right|^{2}\left(y^{2}-1\right) \tilde{R}_{\mathrm{TM}}^{R}\right] .
\end{aligned}
$$

The reflection coefficients $\tilde{R}_{\lambda}^{R}$ are as expressed in (56) but with the wave vectors given by

$$
k_{z \mathrm{i}}=i x E_{j 0} \sqrt{\left(n_{\mathrm{i}}^{2}-1\right) y^{2}+1}, \quad n_{\mathrm{i}}=\left\{1, n_{1}, n_{\mathrm{s}}\right\}
$$

Note that even though the wave vector is imaginary, the final result is a real number, as it should be, because the Fresnel coefficients contain only ratios of wave vectors.

\section{B. Excited atoms}

As mentioned previously, the energy-level shift of an excited atom is also given by Eq. (54). However, one needs to take account of the fact that the quantity $E_{j i} \equiv E_{j}-E_{i}$ can now become negative for $E_{j}<E_{i}$, so that the denominator originating from perturbation theory contributes additional poles lying on the path of $k_{z}$ integration, shown in Fig. 3 and is now to be understood as a Cauchy principal value. These poles are located at $k_{z}= \pm \sqrt{E_{j i}^{2}-\mathbf{k}_{\|}^{2}}$, though their precise location depends on the value of $\left|\mathbf{k}_{\|}\right|$that is not fixed but varies as we carry out the $\mathbf{k}_{\|}$integrations in Eq. (54). For $\left|\mathbf{k}_{\|}\right| \in\left[0,\left|E_{j i}\right|\right]$ the poles are located on the real $k_{z}$ axis but as we increase the value of $\left|\mathbf{k}_{\|}\right|$to exceed $\left|E_{j i}\right|$ both poles move onto the positive imaginary axis according to the convention that $\operatorname{Im}\left(k_{z}\right)>0$. For $\left|\mathbf{k}_{\|}\right|$belonging to the interval $\left[\left|E_{j i}\right|, n_{\mathrm{s}}\left|E_{j i}\right|\right]$ the poles are located on the opposite sides of the branch cut due to the $k_{z \mathrm{~s}}$ and care needs to be taken when evaluating those pole contributions. To evaluate the Cauchy principal value of the $k_{z}$ integral we circumvent the poles and close the contour in the upper half plane, as was done in the previous section. The contribution from the large semicircle vanishes and Eq. (54) acquires pole contributions that are easily worked out by the residue theorem. The energy shift splits into the a "nonresonant" ground-state-like part $\Delta E_{i}$ and a "resonant" oscillatory part $\Delta E_{i}^{\text {res }}$ that arises only if the atom is in an excited state. In analogy to the result of the previous section, the nonresonant part is given by

$$
\begin{aligned}
\Delta E_{i}= & \frac{1}{8 \pi^{2} \epsilon_{0}} \sum_{j \neq i} E_{j i}^{3} \int_{0}^{\infty} d x x^{3} \int_{0}^{1} d y \frac{e^{-2\left|E_{j i}\right| \mathcal{Z} x}}{1+x^{2} y^{2}} \\
& \times\left[\left|\mu_{\|}\right|^{2}\left(y^{2} \tilde{R}_{\mathrm{TE}}^{R}-\tilde{R}_{\mathrm{TM}}^{R}\right)+2\left|\mu_{\perp}\right|^{2}\left(y^{2}-1\right) \tilde{R}_{\mathrm{TM}}^{R}\right],
\end{aligned}
$$

with wave vectors expressed as

$$
k_{z \mathrm{i}}=i x\left|E_{j i}\right| \sqrt{\left(n_{\mathrm{i}}^{2}-1\right) y^{2}+1}, \quad n_{\mathrm{i}}=\left\{1, n_{1}, n_{\mathrm{s}}\right\},
$$

whereas the resonant part is given by

$$
\begin{aligned}
\Delta E_{i}^{\mathrm{res}}= & \operatorname{Re} \frac{i}{8 \pi \epsilon_{0}} \sum_{j<i}\left|E_{j i}\right|^{3} \int_{0}^{\infty} \frac{d q q}{\sqrt{1-q^{2}}} e^{2 i\left|E_{j i}\right| \sqrt{1-q^{2}} \mathcal{Z}} \\
& \times\left\{\left|\mu_{\|}\right|^{2}\left[\left(1-q^{2}\right) \tilde{R}_{\mathrm{TM}}^{R}-\tilde{R}_{\mathrm{TE}}^{R}\right]-2\left|\mu_{\perp}\right|^{2} q^{2} \tilde{R}_{\mathrm{TM}}^{R}\right\},
\end{aligned}
$$

with $q=k_{\|} /\left|E_{j i}\right|$ and

$$
k_{z \mathrm{i}}=\left|E_{j i}\right| \sqrt{n_{\mathrm{i}}^{2}-q^{2}}, \quad n_{\mathrm{i}}=\left\{1, n_{1}, n_{\mathrm{s}}\right\} .
$$

The reflection coefficients are as given in (56). The integral in Eq. (61) contains poles because the dispersion relation present in the denominators of the reflection coefficients has now solutions on the real axis when $q \in\left[n_{\mathrm{s}}, n_{1}\right]$. This signals contributions from surface excitations (trapped modes). This fact has been mentioned in [2], where the interaction of an excited atom with layered dielectric has been studied, although using mainly numerical analysis. Here we attempt to study the results (59) and (61) analytically. To do so it will prove beneficial to rewrite Eq. (61) slightly. We change variables according to $\sqrt{1-q^{2}}=\eta$ and split the contributions to Eq. (61) into two parts. The first one is a contribution from the traveling modes and given by

$$
\begin{aligned}
\Delta E_{i}^{\mathrm{res}, \text { trav }}= & -\operatorname{Re} \frac{i}{8 \pi \epsilon_{0}} \sum_{j<i}\left|E_{j i}\right|^{3} \int_{0}^{1} d \eta e^{2 i\left|E_{j i}\right| \mathcal{Z} \eta} \\
& \times\left\{\left|\mu_{\|}\right|^{2}\left[\tilde{R}_{\mathrm{TE}}^{R}-\eta^{2} \tilde{R}_{\mathrm{TM}}^{R}\right]+2\left|\mu_{\perp}\right|^{2}\left(1-\eta^{2}\right) \tilde{R}_{\mathrm{TM}}^{R}\right\},
\end{aligned}
$$

where the wave vectors in reflection coefficients are all real and can be expressed as

$$
k_{z \mathrm{i}}=\left|E_{j i}\right| \sqrt{n_{\mathrm{i}}^{2}-1+\eta^{2}}, \quad n_{\mathrm{i}}=\left\{1, n_{1}, n_{\mathrm{s}}\right\},
$$

and the second is a contribution from the evanescent modes

$$
\begin{aligned}
\Delta E_{i}^{\mathrm{res}, \text { evan }}= & -\operatorname{Re} \frac{1}{8 \pi \epsilon_{0}} \sum_{j<i}\left|E_{j i}\right|^{3} \int_{0}^{\infty} d \eta e^{-2\left|E_{j i}\right| \mathcal{Z} \eta} \\
& \times\left\{\left|\mu_{\|}\right|^{2}\left[\tilde{R}_{\mathrm{TE}}^{R}+\eta^{2} \tilde{R}_{\mathrm{TM}}^{R}\right]+2\left|\mu_{\perp}\right|^{2}\left(1+\eta^{2}\right) \tilde{R}_{\mathrm{TM}}^{R}\right\},
\end{aligned}
$$

where the wave vectors in reflection coefficients can be expressed as

$$
k_{z \mathrm{i}}=\left|E_{j i}\right| \sqrt{n_{\mathrm{i}}^{2}-1-\eta^{2}}, \quad n_{\mathrm{i}}=\left\{1, n_{1}, n_{\mathrm{s}}\right\} .
$$

Finally, it is worth noting that the imaginary part of Eq. (61) is actually proportional to the modified decay rates [9]. These have already been studied in [16], so we focus on energy shifts only. However, the methods of analysis that are reported in the 
next section do allow one to write down at once equivalent analytical formulas for the decay rates.

\section{ASYMPTOTIC ANALYSIS}

The interaction between the atom and the dielectric is electromagnetic in nature and it is mediated by photons. The atomic system in state $|i\rangle$ evolves in time with a characteristic time scale that is proportional to $E_{j i}^{-1}$, with $E_{j i}$ being the energy-level spacing between the states $|i\rangle$ and $|j\rangle$ which are connected by the strongest dipole transition from state $|i\rangle$. Since it takes a finite time for the photon to make a round trip between the atom and the surface, the atom will have changed by the time the photon comes back. Therefore, the ratio of the time needed by the photon to travel to the surface and back and the typical time scale of atomic evolution is a fundamental quantity that plays a decisive role in characterizing the interaction. In natural units, if $2 E_{j i} \mathcal{Z} \ll 1$ we can safely assume that the interaction is instantaneous and we are in the so-called nonretarded or van der Waals regime. If $2 E_{j i} \mathcal{Z} \gg 1$ the interaction becomes manifestly retarded as the atom will have changed significantly by the time the photon comes back. However, the problem we have considered here provides us with yet another length scale, namely, the thickness of the top layer $L$. We now consider the energy shift in various asymptotic regimes.

\section{A. Ground-state atoms: Electrostatic limit $\left(2 E_{j i} \mathcal{Z} \ll 1\right)$}

In this limit the interaction is instantaneous (or electrostatic) in nature and the energy shift is obtainable using the Green's function of the classical Laplace equation (cf., e.g., [20]). This classical derivation is outlined in Appendix B. The end result for the energy shift reads

$$
\begin{aligned}
\Delta E^{\mathrm{el}}= & -\frac{1}{16 \pi \epsilon_{0}}\left(\left\langle\mu_{\|}^{2}\right\rangle+2\left\langle\mu_{\perp}^{2}\right\rangle t\right) \int_{0}^{\infty} d k k^{2} e^{-2 k \mathcal{Z}} \\
& \times\left(\frac{\frac{n_{1}^{2}-1}{n_{1}^{2}+1}-\frac{n_{1}^{2}-n_{\mathrm{s}}^{2}}{n_{\mathrm{s}}^{2}+n_{1}^{2}} e^{-2 k L}}{1-\frac{n_{1}^{2}-1}{n_{1}^{2}+1} \frac{n_{1}^{2}-n_{\mathrm{s}}^{2}}{n_{\mathrm{s}}^{2}+n_{1}^{2}} e^{-2 k L}}\right),
\end{aligned}
$$

with $\left\langle\mu_{\|}^{2}\right\rangle \equiv\left\langle\mu_{x}^{2}\right\rangle+\left\langle\mu_{y}^{2}\right\rangle$ and $\left\langle\mu_{\perp}^{2}\right\rangle \equiv\left\langle\mu_{z}^{2}\right\rangle$. We now show that one can also obtain the preceding result as a limiting case of the results of previous section, thus providing a cross-check for our general calculation. To start with we note that Eq. (58) cannot be used to take the electrostatic limit in which we mathematically let $E_{j i} \rightarrow 0$ because it has been scaled with $E_{j i}$. Therefore, it is best to start from Eq. (54). The result of Eq. (66) can be derived very quickly if we observe that in the limit $E_{j i} \rightarrow 0$ the branch cut due to $\omega=\sqrt{\mathbf{k}_{\|}^{2}+k_{\mathrm{z}}^{2}}$ is no longer present and the contour in Fig. 4 collapses to a simple enclosure of the point $k_{z}=i\left|\mathbf{k}_{\|}\right|$. The contribution from the TE mode vanishes as the product of the polarization vectors is regular at $k_{z}=i\left|\mathbf{k}_{\|}\right|$, but for the TM mode this point is a simple pole [cf. Eq. (19)]. Therefore, we obtain

$$
\begin{aligned}
\Delta E^{\mathrm{el}}= & -\frac{1}{(2 \pi)^{3} 2 \epsilon_{0}} \sum_{m} \sum_{j \neq 0}\left|\mu_{m}\right|^{2} \int d \mathbf{k}_{\|} \\
& \times 2 \pi i \lim _{k_{z} \rightarrow i\left|\mathbf{k}_{\|}\right|}\left(k_{z}-i\left|\mathbf{k}_{\|}\right|\right) R_{\mathrm{TM}}^{R} e_{\mathrm{TM}}^{m}\left(\mathbf{k}^{+}\right) e_{\mathrm{TM}}^{m}\left(\mathbf{k}^{-}\right) e^{2 i k_{z} z_{0}} .
\end{aligned}
$$

Taking the limit and expressing the remaining integrals in polar coordinates, where the angle integral is elementary, yields Eq. (66) with $\left\langle\mu_{m}^{2}\right\rangle \equiv \sum_{j \neq i}\left|\left\langle i\left|\mu_{m}\right| j\right\rangle\right|^{2}=\left\langle i\left|\mu_{m}^{2}\right| i\right\rangle$. Equation (66) can be further analyzed depending on the relative values of $L$ and $\mathcal{Z}$.

\section{Thin layer $(\mathcal{Z} / L \gg 1)$}

In this case the distance of the atom from the surface is much greater than the thickness of the layer of refractive index $n_{1}$ (but still small enough for the retardation to be neglected). Then, rescaling the integral in Eq. (66) with $k=x / L$ allows us to use Watson's lemma ${ }^{1}$ to derive

$$
\begin{aligned}
\Delta E^{\mathrm{el}} \approx & \Delta E_{n_{\mathrm{s}}}^{\mathrm{el}}-\frac{1}{64 \pi \epsilon_{0} \mathcal{Z}^{3}}\left(\left\langle\mu_{\|}^{2}\right\rangle+2\left\langle\mu_{\perp}^{2}\right\rangle\right) \\
& \times\left[a_{1} \frac{L}{\mathcal{Z}}+a_{2} \frac{L^{2}}{\mathcal{Z}^{2}}+O\left(\frac{L^{3}}{\mathcal{Z}^{3}}\right)\right],
\end{aligned}
$$

with the coefficients $a_{i}$ given by

$$
\begin{gathered}
a_{1}=\frac{3}{n_{1}^{2}} \frac{n_{1}^{4}-n_{\mathrm{s}}^{4}}{\left(n_{\mathrm{s}}^{2}+1\right)^{2}} \\
a_{2}=-\frac{6}{n_{1}^{4}} \frac{\left(n_{1}^{4}-n_{\mathrm{s}}^{4}\right)\left(n_{\mathrm{s}}^{2}+n_{1}^{4}\right)}{\left(n_{\mathrm{s}}^{2}+1\right)^{3}},
\end{gathered}
$$

where $\Delta E_{n_{\mathrm{s}}}^{\mathrm{el}}$ is the well-known electrostatic interaction energy between an atom and a dielectric half space of refractive index $n_{\mathrm{s}}$ that can be obtained by the method of images:

$$
\Delta E_{n_{\mathrm{s}}}^{\mathrm{el}}=-\frac{1}{64 \pi \epsilon_{0} \mathcal{Z}^{3}} \frac{n_{\mathrm{s}}^{2}-1}{n_{\mathrm{s}}^{2}+1}\left(\left\langle\mu_{\|}^{2}\right\rangle+2\left\langle\mu_{\perp}^{2}\right\rangle\right) .
$$

The corrections to this result are represented by the remaining elements of the asymptotic series. Note that if $n_{1}>n_{\mathrm{s}}$, then $a_{1}>0$ and, not surprisingly, the interaction, as compared to a half-space alone, is enhanced by the presence of the thin dielectric layer of higher refractive index $n_{1}$.

\section{Thick layer $(\mathcal{Z} / L \ll 1)$}

In this case the thickness of the layer is much greater than the distance between the atom and the surface. The top layer now appears from the point of view of the atom almost as a half-space of refractive index $n_{1}$ only that it is in fact of finite thickness. To analyze the result (66) in this limit we cast it in a somewhat different form. Note that, especially when $k L$ is large but not only then,

$$
\frac{n_{1}^{2}-1}{n_{1}^{2}+1} \frac{n_{1}^{2}-n_{\mathrm{s}}^{2}}{n_{\mathrm{s}}^{2}+n_{1}^{2}} e^{-2 k L}<1
$$

and the denominator of the integrand in Eq. (66) can be written as geometrical series. Since the series is absolutely convergent

\footnotetext{
${ }^{1}$ The essential idea is to spot that, since the integrand is strongly damped by the exponential, most of the contributions to the integral will come from small values of $k$. Thus, it is permissible to Taylor expand the remaining part of the integrand about $k=0$. For a more rigorous treatment, see [21].
} 
we can integrate it term by term and obtain the following representation of the electrostatic result:

$$
\begin{aligned}
\Delta E^{\mathrm{el}}= & \Delta E_{n_{1}}^{\mathrm{el}}+\frac{1}{16 \pi \epsilon_{0}}\left(\left\langle\mu_{\|}^{2}\right\rangle+2\left\langle\mu_{\perp}^{2}\right\rangle\right) \frac{n_{1}^{2}}{n_{1}^{4}-1} \\
& \times \sum_{\nu=1}^{\infty}\left(\frac{n_{1}^{2}-1}{n_{1}^{2}+1} \frac{n_{1}^{2}-n_{\mathrm{s}}^{2}}{n_{\mathrm{s}}^{2}+n_{1}^{2}}\right)^{\nu} \frac{1}{(\mathcal{Z}+\nu L)^{3}},
\end{aligned}
$$

where $\Delta E_{n_{1}}^{\mathrm{el}}$ is the electrostatic energy shift due to a single half space of refractive index $n_{1}$, that is, Eq. (68) with $n_{\mathrm{s}}$ replaced with $n_{1}$. The sum in Eq. (70) represents the correction to $\Delta E_{n_{1}}^{\mathrm{el}}$ due to the finite thickness of the layer. For fixed $\mathcal{Z}$ and $L$, it can be easily computed numerically to any desired degree of accuracy. We note, however, that to the leading order in $\mathcal{Z} / L$ the interaction is weakened by the same amount independently of the distance of the atom from the surface and therefore is not measurable. The next-to-leading order correction is the first to be distance dependent and is proportional to $\mathcal{Z} / L^{4}$, which can be easily seen by expanding the factor in series around $\mathcal{Z} / \nu L=0$ :

$$
\frac{1}{(\mathcal{Z}+v L)^{3}} \approx \frac{1}{v^{3} L^{3}}-\frac{3 Z}{v^{4} L^{4}}+O\left(\frac{Z^{2}}{L^{5}}\right) .
$$

\section{B. Ground-state atoms: Retarded limit $\left(2 \mathcal{Z} E_{j i} \gg 1\right)$}

\section{Thin layer $(\mathcal{Z} / L \gg 1)$}

In this case we study the situation when the top layer is much thinner than the distance between the atom and the surface. To obtain the asymptotic series we use Watson's lemma in much the same way as in the electrostatic case [21]. Series expansion of the integrand in Eq. (58) about $x=0$ decouples the integrals and the resulting integrals can be calculated analytically. Thus, to first approximation, for an atom located sufficiently far from the interface, the impact of the thin dielectric layer on the standard Casimir-Polder interaction can be described by

$$
\begin{aligned}
\Delta E^{\mathrm{ret}}= & \Delta E_{n_{\mathrm{s}}}^{\mathrm{ret}}-\frac{1}{16 \pi^{2} \epsilon_{0} \mathcal{Z}^{4}} \sum_{j \neq 0}\left[\frac{a_{\|}\left|\mu_{\|}\right|^{2}+2 a_{\perp}\left|\mu_{\perp}\right|^{2}}{E_{j 0}}\right] \frac{L}{\mathcal{Z}} \\
& +O\left(\frac{L^{2}}{\mathcal{Z}^{2}}\right),
\end{aligned}
$$

where $\Delta E_{n_{\mathrm{s}}}^{\mathrm{ret}}$ is the retarded limit of energy shift as caused by a single dielectric half space of refractive index $n_{\mathrm{s}}$, which was calculated in [9]. We give this result in Appendix C. The coefficients $a_{\|}$and $a_{\perp}$ in (72) can be expressed in terms of elementary functions as

$$
\begin{aligned}
a_{\|}= & \frac{1}{n_{1}^{2}} \frac{n_{1}^{2}-n_{\mathrm{s}}^{2}}{\left(n_{\mathrm{s}}^{2}-1\right)^{2}\left(n_{\mathrm{s}}^{2}+1\right)}\left[n_{\mathrm{s}}^{5}\left(6 n_{\mathrm{s}}-3\right)\left(n_{\mathrm{l}}^{2}-1\right)\right. \\
& \left.+3 n_{\mathrm{s}}^{2}\left(n_{1}^{2}+1\right)-n_{1}^{2}\left(2 n_{\mathrm{s}}^{4}+3 n_{\mathrm{s}}^{3}+3 n_{\mathrm{s}}-8\right)\right] \\
& -\frac{n_{1}^{2}-n_{\mathrm{s}}^{2}}{n_{1}^{2}\left(n_{\mathrm{s}}^{2}-1\right)^{5 / 2}} \ln \left(\sqrt{n_{\mathrm{s}}^{2}-1}+n_{\mathrm{s}}\right)\left[2 n_{\mathrm{s}}^{2} n_{1}^{2}\left(n_{\mathrm{s}}^{2}-1\right)^{2}\right. \\
& \left.-2 n_{\mathrm{s}}^{4}\left(n_{\mathrm{s}}^{2}-1\right)+n_{1}^{2}\right]-\frac{n_{\mathrm{s}}^{4}}{2 n_{1}^{2}} \frac{n_{1}^{2}-n_{\mathrm{s}}^{2}}{\left(n_{\mathrm{s}}^{2}-1\right)^{2}\left(n_{\mathrm{s}}^{2}+1\right)^{3 / 2}}
\end{aligned}
$$

$$
\begin{aligned}
& \times \ln \left(\frac{\sqrt{n_{\mathrm{s}}^{2}+1}+1}{\sqrt{n_{\mathrm{s}}^{2}+1}-1} \frac{\sqrt{n_{\mathrm{s}}^{2}+1}-n_{\mathrm{s}}}{\sqrt{n_{\mathrm{s}}^{2}+1}+n_{\mathrm{s}}}\right) \\
& \times\left[2 n_{\mathrm{s}}^{4}\left(n_{1}^{2}-1\right)-2 n_{\mathrm{s}}^{2}-3 n_{1}^{2}+1\right] \\
a_{\perp}= & \frac{1}{n_{1}^{2}} \frac{n_{1}^{2}-n_{\mathrm{s}}^{2}}{\left(n_{\mathrm{s}}^{2}-1\right)^{2}\left(n_{\mathrm{s}}^{2}+1\right)}\left[n_{\mathrm{s}}^{4}\left(4 n_{\mathrm{s}}^{2}-3 n_{\mathrm{s}}-3\right)-n_{\mathrm{s}}^{2}\left(12 n_{\mathrm{s}}^{6}\right.\right. \\
& \left.\left.-6 n_{\mathrm{s}}^{5}+2\right)\left(n_{1}^{2}-1\right)+n_{1}^{2}\left(2 n_{\mathrm{s}}^{6}+7 n_{\mathrm{s}}^{4}-3 n_{\mathrm{s}}^{3}+2\right)\right] \\
& +\frac{n_{\mathrm{s}}^{2}}{n_{1}^{2}} \frac{n_{1}^{2}-n_{\mathrm{s}}^{2}}{\left(n_{\mathrm{s}}^{2}-1\right)^{5 / 2}} \ln \left(\sqrt{n_{\mathrm{s}}^{2}-1}+n_{\mathrm{s}}\right)\left[n _ { 1 } ^ { 2 } \left(4 n_{\mathrm{s}}^{6}-6 n_{\mathrm{s}}^{4}\right.\right. \\
& \left.\left.+3 n_{\mathrm{s}}^{2}-1\right)-n_{\mathrm{s}}^{2}\left(2 n_{\mathrm{s}}^{2}-1\right)^{2}\right]+\frac{n_{\mathrm{s}}^{6}}{2 n_{1}^{2}} \frac{n_{1}^{2}-n_{\mathrm{s}}^{2}}{\left(n_{\mathrm{s}}^{2}-1\right)^{2}\left(n_{\mathrm{s}}^{2}+1\right)^{3 / 2}} \\
& \times \ln \left(\frac{\sqrt{n_{\mathrm{s}}^{2}+1}+1}{\sqrt{n_{\mathrm{s}}^{2}+1}-1} \frac{\sqrt{n_{\mathrm{s}}^{2}+1}-n_{\mathrm{s}}}{\sqrt{n_{\mathrm{s}}^{2}+1}+n_{\mathrm{s}}}\right) \\
& \times\left[4 n_{\mathrm{s}}^{4}\left(n_{1}^{2}-1\right)+2 n_{\mathrm{s}}^{2}\left(n_{1}^{2}-2\right)-3 n_{1}^{2}+1\right] .
\end{aligned}
$$

Both $a_{\|}$and $a_{\perp}$ are positive for $n_{1}>n_{\mathrm{s}}$ so that, as one would expect, the interaction, as compared to a half space alone, is enhanced by the thin dielectric layer of the higher refractive index $n_{1}$. The preceding result simplifies significantly in the case when $n_{\mathrm{s}}$ approaches unity, that is, when the situation resembles that of an atom interacting with a dielectric slab of refractive index $n_{1}$. The coefficients $a_{\|}$and $a_{\perp}$ reduce then to those recently calculated in [1] and are given by

$$
\begin{aligned}
& a_{\|}=\frac{\left(n_{1}^{2}-1\right)\left(9 n_{1}^{2}+5\right)}{10 n_{1}^{2}}, \\
& a_{\perp}=\frac{\left(n_{1}^{2}-1\right)\left(5 n_{1}^{2}+4\right)}{10 n_{1}^{2}} .
\end{aligned}
$$

\section{Thick layer $(\mathcal{Z} / L \ll 1)$}

Here we assume that the thickness of the top layer is much greater than the distance between the atom and the surface, but is still large enough for retardation to occur. Note that the reflection coefficient $\tilde{R}_{\lambda}^{R}(22)$ can be separated into $L$ dependent and $L$-independent parts in the following manner:

$$
\tilde{R}_{\lambda}^{R}=r_{\lambda}^{\mathrm{vl}}+\frac{\left[1-\left(r_{\lambda}^{\mathrm{vl}}\right)^{2}\right] r_{\lambda}^{\mathrm{ls}} e^{2 i k_{z 1} L}}{1+r_{\lambda}^{\mathrm{vl}} r_{\lambda}^{1 \mathrm{~s}} e^{2 i L k_{z 1} L}}
$$

This way of writing the reflection coefficient splits the energy shift (58) into a shift due to the single interface of refractive index $n_{1}$ and corrections due to the finite thickness and the underlying material. It can be shown numerically (see Sec. V) that for large values of $L$ the correction term is vanishingly small and can be safely discarded. Brute-force asymptotic analysis allows us to draw similar conclusions as in the electrostatic case, Sec. IV A 2. To leading order the interaction gets altered by the same amount regardless of the position of the atom with respect to the interface. The next-to-leading-order correction is proportional to $\mathcal{Z} / L^{5}$. 


\section{Excited atoms: Nonretarded limit $\left(2 \mathcal{Z}\left|E_{j i}\right| \ll 1\right)$}

The energy shift of an excited atom is given by Eqs. (59) and (61). The nonresonant part, that is, Eq. (59), has the same form as the energy shift of the ground-state atom and has been analyzed in the previous section. Therefore, we now focus on the resonant part of the interaction that is given by Eq. (61). In order to conveniently obtain the nonretarded limit of (61) we work with its slightly modified form given in Eqs. (62) and (64).

We start by noting that close to the interface we expect asymptotic series to be in the inverse powers of $\mathcal{Z}$. Eq. (62), where the $\eta$ integration runs over $\eta \in[0,1]$, contributes only positive powers of $\mathcal{Z}$. This is most easily seen by expanding the exponential $\exp \left(2 i\left|E_{j i}\right| \mathcal{Z} \eta\right)$ about origin as we may do in the limit $2 \mathcal{Z}\left|E_{j i}\right| \rightarrow 0$. Therefore, to leading-order in the electrostatic limit, only (64) contributes. Further we analyze (64) by setting $\eta=\beta /\left(\left|E_{j i}\right| \mathcal{Z}\right)$. Then, according to (65), in the limit $\left|E_{j i}\right| \mathcal{Z} \rightarrow 0$ the wave vectors can effectively be approximated as

$$
k_{z} \approx k_{z 1} \approx k_{z \mathrm{~s}} \approx i \frac{\beta}{\mathcal{Z}}
$$

Then the result for the energy shift, after substituting $\beta=k \mathcal{Z}$, reduces to

$$
\begin{aligned}
\Delta E^{\mathrm{res}, \mathrm{el}}= & -\frac{1}{8 \pi \epsilon_{0}} \sum_{j<i}\left(\left|\mu_{\|}\right|^{2}+2\left|\mu_{\perp}\right|^{2}\right) \int_{0}^{\infty} d k k^{2} e^{-2 k \mathcal{Z}} \\
& \times \frac{\frac{n_{1}^{2}-1}{n_{1}^{2}+1}-\frac{n_{1}^{2}-n_{\mathrm{s}}^{2}}{n_{\mathrm{s}}^{2}+n_{1}^{2}} e^{-2 k L}}{1-\frac{n_{1}^{2}-1}{n_{1}^{2}+1} \frac{n_{1}^{2}-n_{\mathrm{s}}^{2}}{n_{\mathrm{s}}^{2}+n_{1}^{2}} e^{-2 k L}}
\end{aligned}
$$

This result turns out to have the same dependence on $\mathcal{Z}$ and $L$ as the Coulomb interaction of the ground-state atom [cf. Eq. (66)]; therefore, we do not analyze Eq. (75) any further. Note, however, that the dependence on the atomic states is different in Eqs. (66) and (75). We also point out that in the electrostatic limit, to the order we are considering, the quantity $\Delta E^{\text {res,el }}$ turns out to be real, which would imply that the corrections to the decay rates vanish. However, this conclusion is incorrect as it is known that the change of spontaneous emission in the nonretarded limit is in fact constant for a nondispersive dielectric half space [9]. However, any serious analysis of the changes of the decay rates induced by a surface needs to take into account the absorption of the material, which in the nonretarded limit plays a crucial role and cannot be neglected. Furthermore, we note that we have started from Eq. (61), which, as explained before, contains poles on the real axis signaling the trapped modes. However, the denominator of (75) never vanishes, which reflects the fact that in the electrostatic limit the trapped modes cease to exist and do not contribute toward the energy shifts, as first mentioned in [2].

\section{Excited atoms: Retarded limit $\left(2 \mathcal{Z}\left|E_{j i}\right| \gg 1\right)$}

The leading-order behavior of Eq. (61) in the retarded limit can be obtained by repeated integration by parts. Unlike in the electrostatic case, now both equations Eqs. (62) and (64) contribute. We integrate them by parts and note that the nonoscillatory contributions that arise from the boundary terms evaluated at $\eta=0$ cancel out. It turns out that the leading-order contributions to the energy shift are due to the parallel component of the atomic dipole moment. They dominate the retarded interaction energy and behave as $\mathcal{Z}^{-1}$. The contributions due to the component of the atomic dipole moment that is perpendicular to the surface contribute only terms proportional to $\mathcal{Z}^{-2}$. We find that in the retarded limit the interaction energy up to the leading order is given by

$$
\begin{aligned}
\Delta E_{i}^{\mathrm{res}, \mathrm{ret}}= & -\frac{1}{8 \pi \epsilon_{0} \mathcal{Z}} \sum_{j<i}\left|E_{j i}\right|^{2}\left|\mu_{\|}\right|^{2} \\
& \times \frac{1}{1+2 r_{\mathrm{vl}} r_{\mathrm{ls}} \cos \left(2\left|E_{j i}\right| \tau\right)+r_{\mathrm{vl}}^{2} r_{\mathrm{ls}}^{2}} \\
& \times\left\{r_{\mathrm{vl}}\left(1+r_{\mathrm{ls}}^{2}\right) \cos \left(2\left|E_{j i}\right| \mathcal{Z}\right)\right. \\
& +r_{\mathrm{vl}}^{2} r_{\mathrm{ls}} \cos \left[2\left|E_{j i}\right|(\mathcal{Z}-\tau)\right] \\
& \left.+r_{\mathrm{ls}} \cos \left[2\left|E_{j i}\right|(\mathcal{Z}+\tau)\right]\right\},
\end{aligned}
$$

where we have defined the optical thickness of the layer as $\tau=n_{1} L$ and

$$
r_{\mathrm{vl}}=\frac{1-n_{1}}{1+n_{\mathrm{l}}}, \quad r_{\mathrm{ls}}=\frac{n_{1}-n_{\mathrm{s}}}{n_{1}+n_{\mathrm{s}}} .
$$

The final result agrees with that derived for a half-space in [9] if we take either $L \rightarrow 0$ or $n_{1} \rightarrow n_{\mathrm{s}}$, which is a consistency check of our calculation. However, the limit of perfect reflectivity of the top layer does not make sense and one has to start from Eq. (61) and rewrite the reflection coefficient in the form (73) in order to study this case.

Equation (76) is valid only approximately when the distance between the atom and the surface is much greater than the wavelength of the strongest atomic dipole transition, but it nevertheless allows us to draw important conclusions. We note that the interaction is resonant; that is, it is enhanced for certain values of $L E_{j i}$. The most convenient way to understand the essence of these resonance effects is to take the slab limit of Eq. (76), that is, set $n_{\mathrm{s}}=1$. In this limit we have

$$
\begin{aligned}
\Delta E_{i}^{\mathrm{res}, \text { ret }}= & -\frac{1}{8 \pi \epsilon_{0} \mathcal{Z}} \sum_{j<i}\left|E_{j i}\right|^{2}\left|\mu_{\|}\right|^{2} \\
& \times \frac{1}{1-2 r_{\mathrm{vl}}^{2} \cos \left(2\left|E_{j i}\right| \tau\right)+r_{\mathrm{vl}}^{4}} \\
& \times\left\{r_{\mathrm{vl}}\left(1+r_{\mathrm{vl}}^{2}\right) \cos \left(2\left|E_{j i}\right| \mathcal{Z}\right)\right. \\
& -r_{\mathrm{vl}}^{3} \cos \left[2\left|E_{j i}\right|(\mathcal{Z}-\tau)\right] \\
& \left.-r_{\mathrm{vl}} \cos \left[2\left|E_{j i}\right|(\mathcal{Z}+\tau)\right]\right\} .
\end{aligned}
$$

It is easily seen that whenever $\cos \left(2\left|E_{j i}\right| \tau\right)=1$ then $\Delta E_{i}^{\text {res, ret }}=0$; that is, the leading-order interaction vanishes. Conversely, the amplitude of oscillations in Eq. (78) is maximized when $\cos \left(2\left|E_{j i}\right| \tau\right)=-1$. Therefore, we have a condition for resonance in terms of the wavelength of the strongest atomic dipole transition $\lambda_{j i}$,

$$
\tau=n_{1} L=\frac{\lambda_{j i}}{2}\left(\kappa+\frac{1}{2}\right), \quad \kappa=0,1,2 \ldots
$$

Equation (79) holds for $Z\left|E_{j i}\right| \gg 1$ but if the value of $Z\left|E_{j i}\right|$ approaches unity, the relation loses its validity, because complications arise from the fact that when the atom is close to the surface the evanescent waves come into play, whereas 
the condition (79) refers to the interaction of an atom with traveling modes only. In the nonretarded limit $\mathcal{Z}\left|E_{j i}\right| \ll 1$ the notion of resonance loses its meaning altogether [cf. Eq. (75)]. Exploring the extreme case in the retarded limit we note that at antiresonance, that is, when

$$
\tau=n_{1} L=\frac{\lambda_{j i}}{2} \kappa, \quad \kappa=0,1,2 \ldots
$$

Eq. (76) becomes

$$
\Delta E_{i}^{\mathrm{res}, \mathrm{ret}}=\frac{1}{8 \pi \epsilon_{0} \mathcal{Z}} \frac{n_{\mathrm{s}}-1}{n_{\mathrm{s}}+1} \sum_{j<i}\left|E_{j i}\right|^{2}\left|\mu_{\|}\right|^{2} \cos \left(2\left|E_{j i}\right| \mathcal{Z}\right)
$$

that is, the atom does not feel the presence of the layer and the interaction assumes the form of that between an atom and a single half space of refractive index $n_{\mathrm{s}}$ (cf. [9]). This means that in the retarded regime the leading-order interaction between an excited atom and a slab of thickness $L$ vanishes whenever the optical thickness of the slab $\tau=n_{1} L$ is equal to a half-integer multiple of the wavelength of the dominant atomic transition $\lambda_{j i}$ (cf. also Fig. 11 later on). Conversely, at resonance the shift becomes

$$
\Delta E_{i}^{\mathrm{res}, \mathrm{ret}}=\frac{1}{8 \pi \epsilon_{0} \mathcal{Z}} \frac{n_{1}^{2}-n_{\mathrm{s}}}{n_{1}^{2}+n_{\mathrm{s}}} \sum_{j<i}\left|E_{j i}\right|^{2}\left|\mu_{\|}\right|^{2} \cos \left(2\left|E_{j i}\right| \mathcal{Z}\right),
$$

so that the amplitude of oscillations exceeds the amplitude that would have been caused by a single half space of refractive index $n_{1}$. It also reaches the perfect reflector limit $n_{1} \rightarrow \infty$ more rapidly. Finally, we also remark that the meaning of the conditions (79) and (80) is interchanged if the refractive index of the substrate $n_{\mathrm{s}}$ exceeds that of the layer $n_{1}$, that is, when $n_{\mathrm{s}}>n_{1}$.

\section{NUMERICAL EXAMPLES}

In this section we present a few numerical results designed to illustrate the influence of the dielectric layer on the CasimirPolder interaction between an atom and a dielectric half space. In practice, the sum over intermediate states $j$ in Eq. (58) and in Eq. (61) is restricted to one or a few states to which there are strong dipole transitions. Hence, we assume a twolevel system in which $E_{j i}$ is a single number, namely, the energy spacing of the levels with the strongest dipole transition. Additionally, we focus just on the contributions to the energy shift due to the component of the atomic dipole that is parallel to the interface of the dielectrics. The contributions due to the perpendicular components of the atomic dipole moment can be easily generated from Eq. (58) using standard computer algebra packages like MATHEMATICA or MAPLE. We start by simple checks on the asymptotic expansions derived in the previous section.

\section{A. Ground-state atoms}

We choose to plot the energy-level shift $\Delta E$ multiplied by $\mathcal{Z}^{4}$ so that the asymptotic behavior of it as a function of distance is more apparent, because $\mathcal{Z}^{4} \Delta E$ for a dielectric half space approaches constant [9]. Then one can easily track

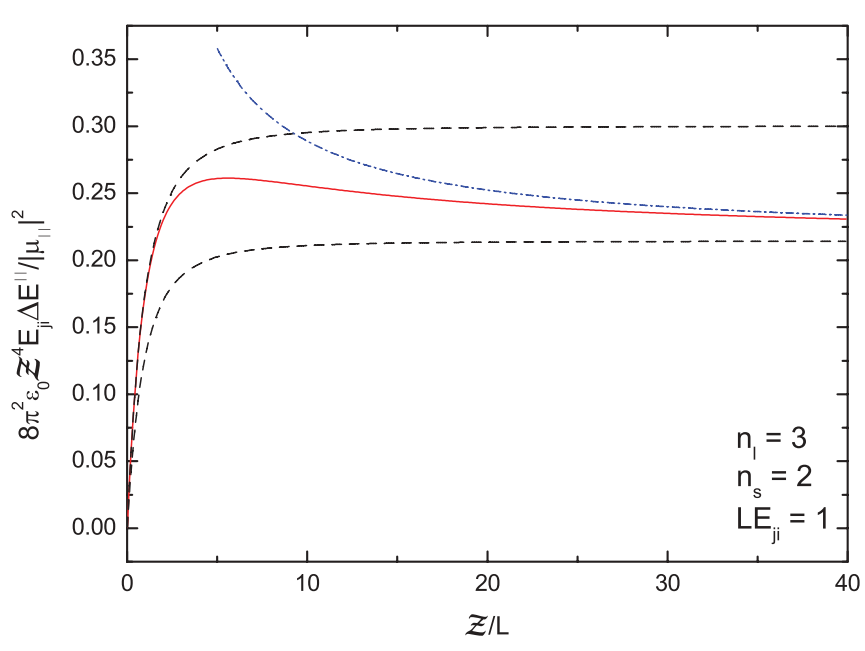

FIG. 5. (Color online) Plot of the exact energy-level shift contributions $\Delta E^{\|}$(solid line) [Eq. (58)] multiplied by $\mathcal{Z}^{4}$. Dashed lines represent the energy shifts due to the single dielectric half-spaces of refractive indices $n_{\mathrm{l}}$ (top) and $n_{\mathrm{s}}$ (bottom), whereas the dotted-dashed line represents the asymptotic approximation (72).

the variation of the energy shift caused by the top layer as compared to the half space shifts (Figs. 5 and 6). We remark that even though the derivation of the energy shift in this paper was based on the assumption $n_{1}>n_{\mathrm{s}}$, the results are also valid in the case when the top layer has a smaller reflectivity than the substrate. In such a case the result can be used, for example, to model a thin layer of oxide or any kind of dirt on the substrate which is often present under realistic conditions.

The asymptotic expansion (72) works well for large $\mathcal{Z} / L$ and not-too-high values of the refractive index $n_{1}$. This is demonstrated in Fig. 7. The increase of the refractive index $n_{1}$ has an impact on the accuracy of the approximation, which is valid provided

$$
\mathcal{Z} \gg \lambda_{j i}+\tau_{1}
$$

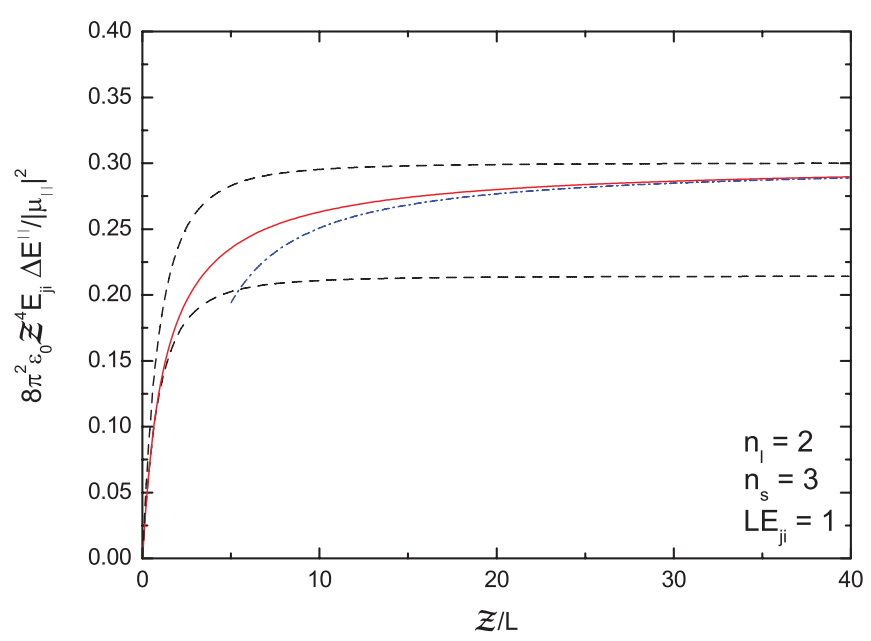

FIG. 6. (Color online) Plot of the exact energy-level shift $\Delta E^{\|}$ (solid line) [Eq. (58)] multiplied by $\mathcal{Z}^{4}$. Dashed lines represent the energy shifts due to the single dielectric half spaces of refractive indices $n_{1}$ (bottom) and $n_{\mathrm{s}}$ (top), whereas the dotted-dashed line represents the asymptotic approximation (72). 


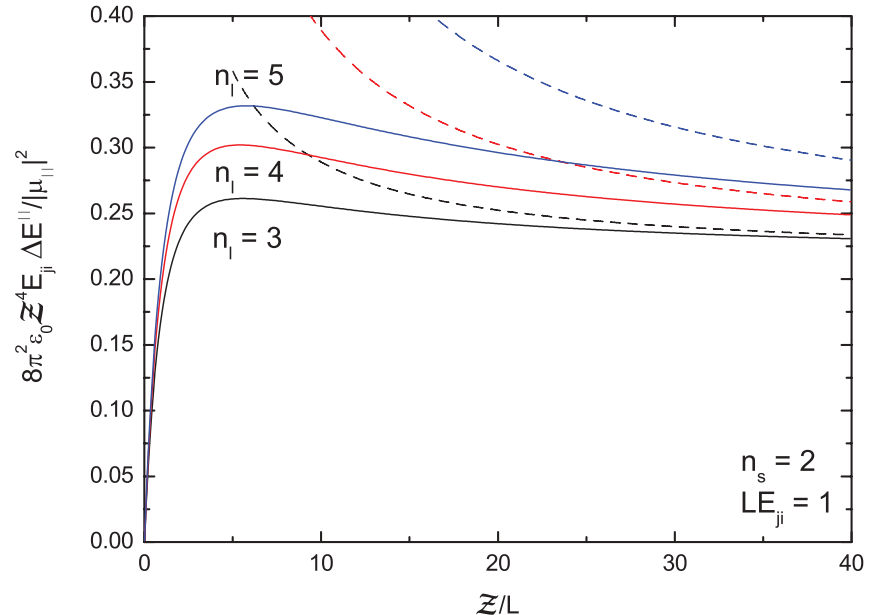

FIG. 7. (Color online) Plot of the exact energy shift $\Delta E^{\|}$, [solid lines, Eq. (58)], multiplied by $\mathcal{Z}^{4}$ together with the asymptotic approximations [dashed lines, Eq. (72)].

with $\lambda_{j i}$ being the wavelength of the dominant atomic transition and $\tau_{1}=n_{1} L$ is the optical thickness of the top layer.

In Fig. 8 we demonstrate the behavior of the energy shift depending on the various values of the parameter $E_{j i}$ measured in units of the layer's thickness. For small $E_{j i}$ we clearly observe linear behavior that corresponds to the $\mathcal{Z}^{-3}$ dependence of the shift in the electrostatic regime.

We also find it instructive to plot the energy-level shift as a function of the thickness of the top layer $L$ for different values of the refractive index $n_{1}$ while keeping the distance of the atom from the surface fixed (Figs. 9 and 10).

\section{B. Excited atoms}

The energy shift of an excited atom splits into two distinct parts [cf. Eqs. (59) and (61)]. The nonoscillatory part displays the same behavior as the energy shift of the ground-state atoms, which we have already analyzed numerically in the previous section. Here we focus on the oscillatory contributions to the

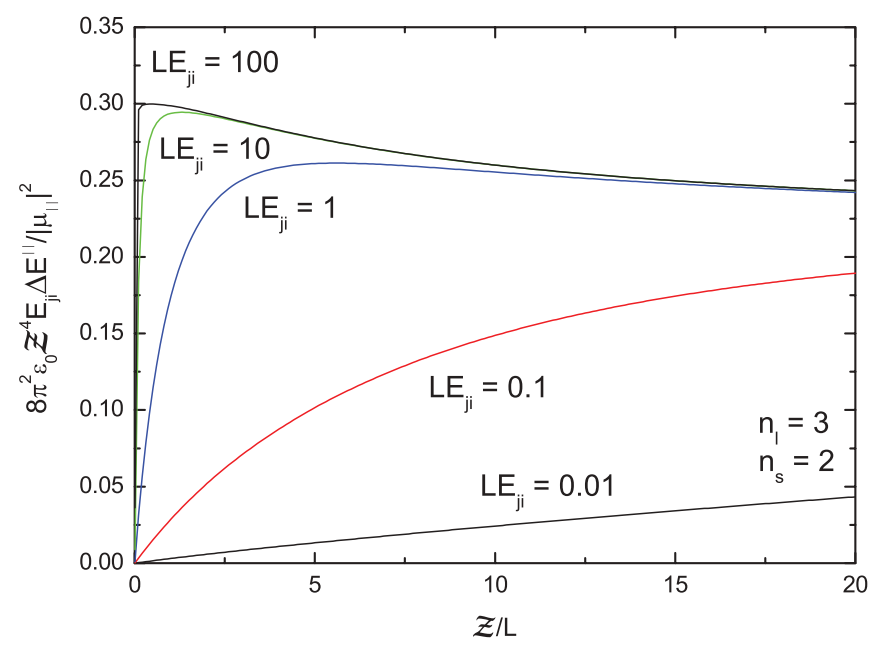

FIG. 8. (Color online) Plot of the exact energy shift $\Delta E^{\|}$ [Eq. (58)] multiplied by $\mathcal{Z}^{4}$ as a function of $\mathcal{Z} / L$ for various values of the retardation parameter $E_{j i} L$.

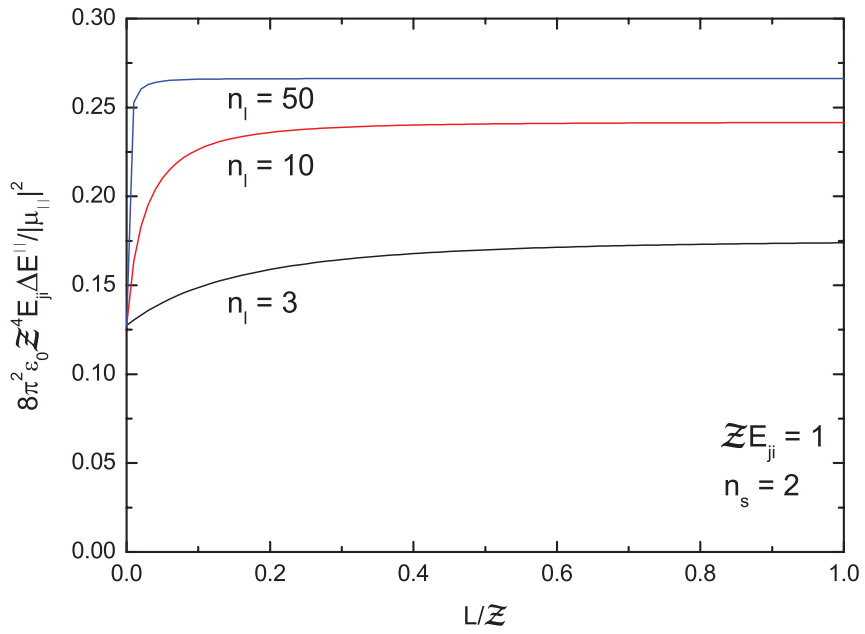

FIG. 9. (Color online) Plot of the exact energy shift $\Delta E^{\|}$ [Eq. (58)] multiplied by $\mathcal{Z}^{4}$ as a function of a layer's thickness $L$ measured in units of fixed atom-wall separation $\mathcal{Z}$ for various values of the layer's refractive index $n_{1}>n_{\mathrm{s}}$.

level shifts that are given by Eq. (61). We choose to plot the dimensionless integrals contained in Eqs. (62) and (64) as this is numerically more efficient than plotting the integral in Eq. (61). It should be borne in mind that the reflection coefficients contain the dispersion relation in denominators that now has solutions on the real axis. For the purpose of the present demonstration it is sufficient to simply displace the poles off the real axis by adding a small imaginary part to the denominator of the reflection coefficients, which amounts to taking the Cauchy principal value during numerical integration.

In Fig. 11 we demonstrate that, indeed, if the antiresonance condition (80) is satisfied, the interaction energy between the excited atom and the slab is strongly suppressed for $\mathcal{Z} E_{j i} \gg 1$. In general, for the layered dielectric rather than the slab, the effect of resonance is shown in Figs. 12 and 13. Note that the energy-level shift in an excited atom due to the layered

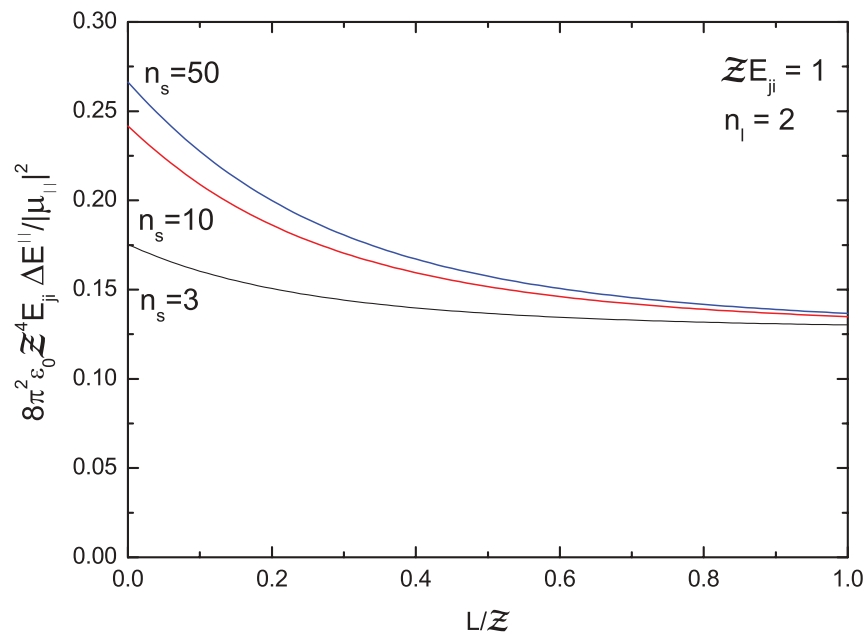

FIG. 10. (Color online) Plot of the exact energy shift $\Delta E^{\|}$ [Eq. (58)] multiplied by $\mathcal{Z}^{4}$ as a function of layer's thickness $L$ measured in units of fixed atom-wall separation $\mathcal{Z}$ for various values of the substrate's refractive index $n_{\mathrm{s}}>n_{\mathrm{l}}$. 


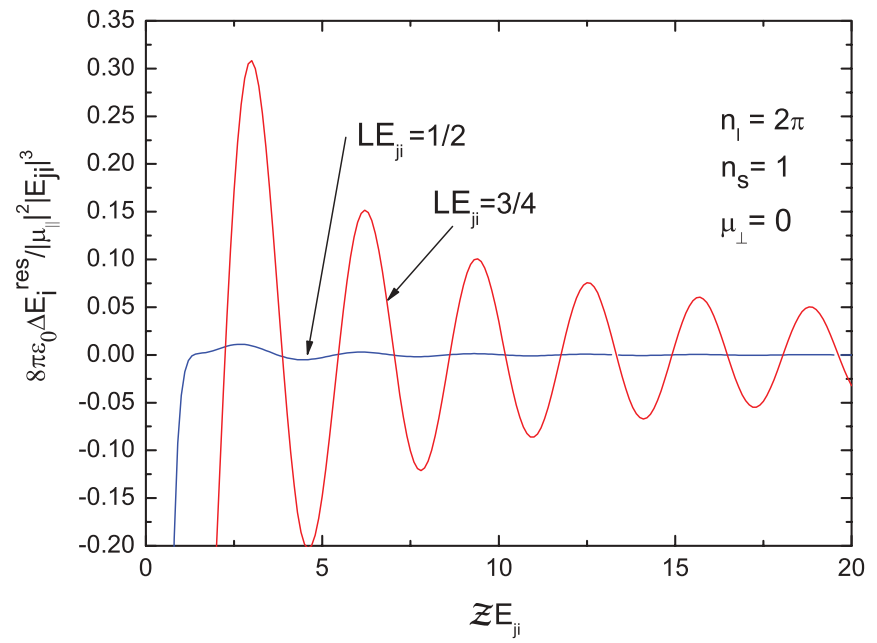

FIG. 11. (Color online) Plot of the exact energy-level shift (61) (resonant part) in an excited atom due to the parallel component of the atomic dipole moment placed in front of a slab of thickness $L$ and refractive index $n_{1}=2 \pi$. The energy spacing of the dominant atomic transition is such that $L E_{j i}=3 / 4$; that is, it satisfies the resonance condition (80). As is seen, when $L E_{j i}=1 / 2$, the energy shift in the retarded regime is strongly suppressed [cf. Eq. (76)].

dielectric can be significantly enhanced. Unlike in the case of the ground-state atom, where the energy shift caused by the layered structure of refractive indices $n_{1}$ and $n_{\mathrm{s}}$ is bounded by the single half-space shifts (compare Fig. 5), the excited atom can experience shifts greater than those caused by the unlayered half space of the refractive index $n=\max \left(n_{1}, n_{\mathrm{s}}\right)$ (Fig. 12), which is due to resonance effects. Conversely, it

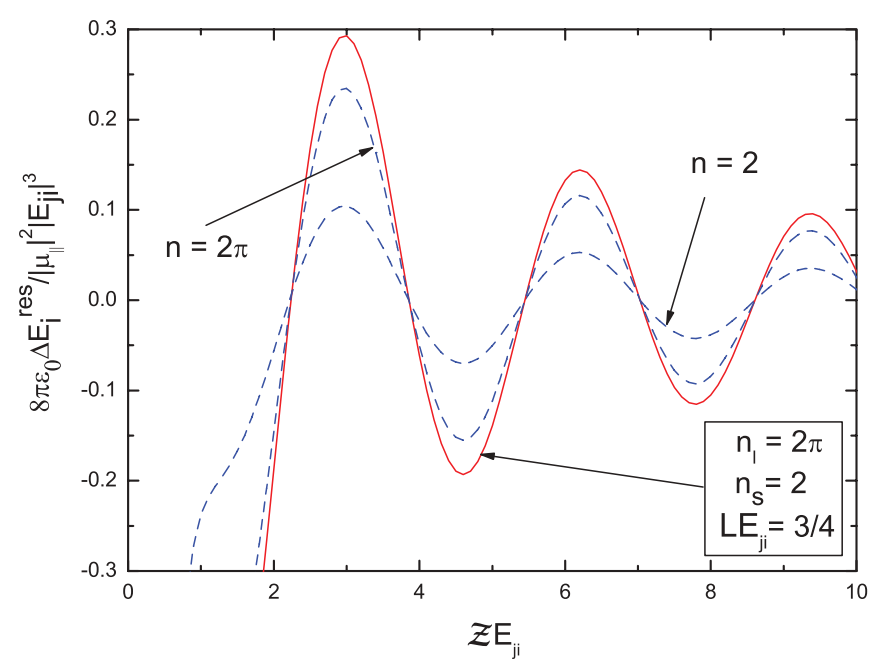

FIG. 12. (Color online) Plot of the exact energy-level shift (61) (resonant part) in an excited atom due to the parallel component of the atomic dipole moment placed in front of the layered dielectric with parameters as shown on the graph (solid line). The resonant condition (79) is satisfied so that the interaction is enhanced. The amplitude of oscillations exceeds the one that would have been caused by an unlayered half space of the refractive index $n=2 \pi$ [cf. Eq. (81)]. Compare also Fig. 5. The dashed lines represent the interaction between an atom and single half space of refractive index $n$ as indicated.

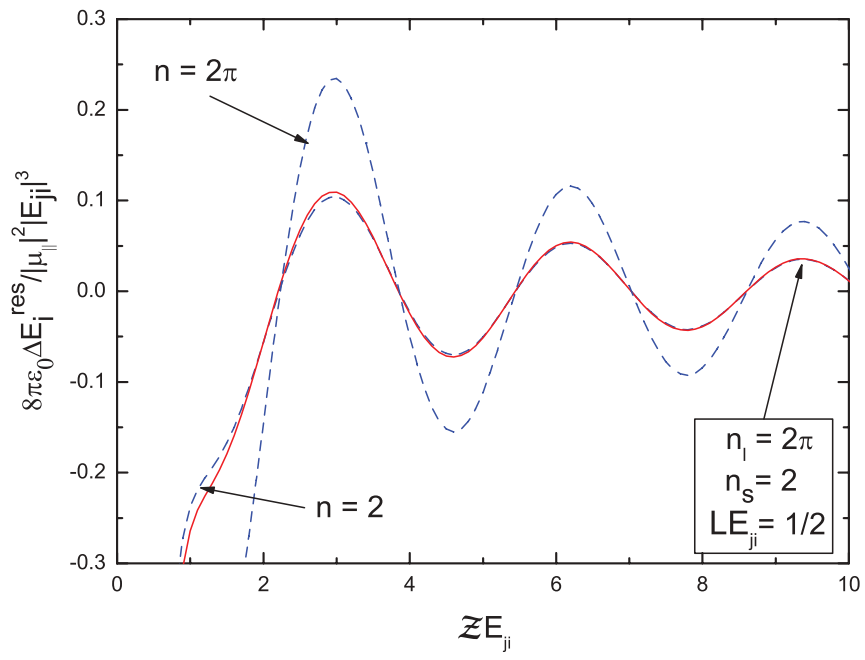

FIG. 13. (Color online) Plot of the exact energy-level shift (61) (resonant part) in an excited atom due to the parallel component of the atomic dipole moment placed in front of the layered dielectric with parameters as shown on the graph (solid line). The antiresonant condition (80) is satisfied so that the presence of the layer is almost unnoticeable[cf. Eq. (81)]. The dashed lines represent the interaction between an atom and single half space of refractive index $n$ as indicated.

is also possible that the interaction with the layer will be unnoticeable if the antiresonance condition (80) is satisfied (Fig. 13). Next, in Fig. 14, we show that the approximation of Eq. (61) derived in (76) turns out to be quite accurate and can be safely used to quickly estimate the energy shift in an excited atom caused by the layered dielectric, provided the condition $\mathcal{Z} E_{j i} \gg 1$ is satisfied. It is also interesting to plot the resonant part of the energy shift as a function of $L E_{j i}$ while keeping $Z E_{j i}$ fixed. This is done in Fig. 15. It is seen that the energy shift indeed experiences the oscillatory resonant behavior. The subsequent minima and maxima are less and

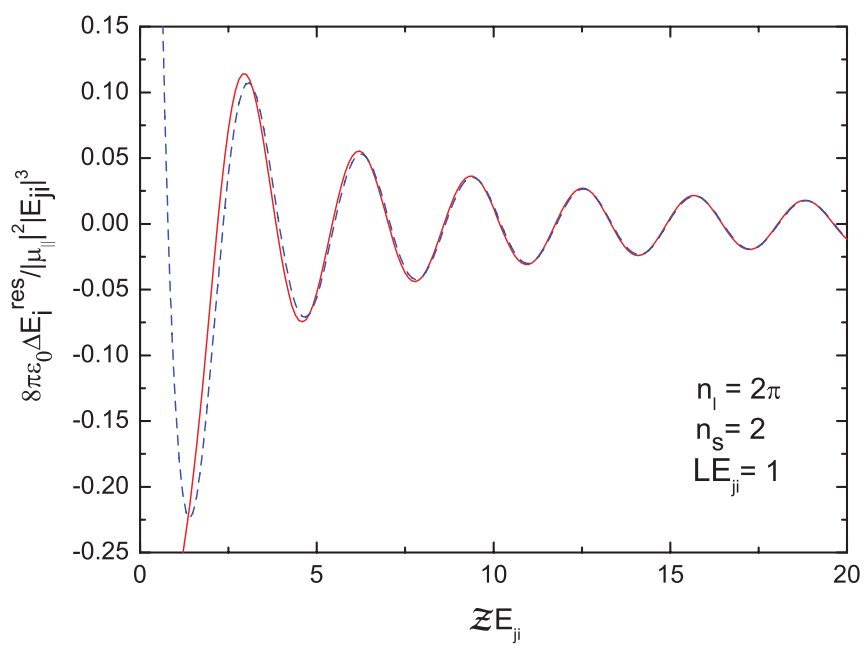

FIG. 14. (Color online) Plot of the exact energy-level shift (61) (resonant part) in an excited atom due to the parallel component of the atomic dipole moment placed in front of the layered dielectric with parameters as shown on the graph (solid line). The dashed line represents the approximation in the retarded regime, Eq. (76). 


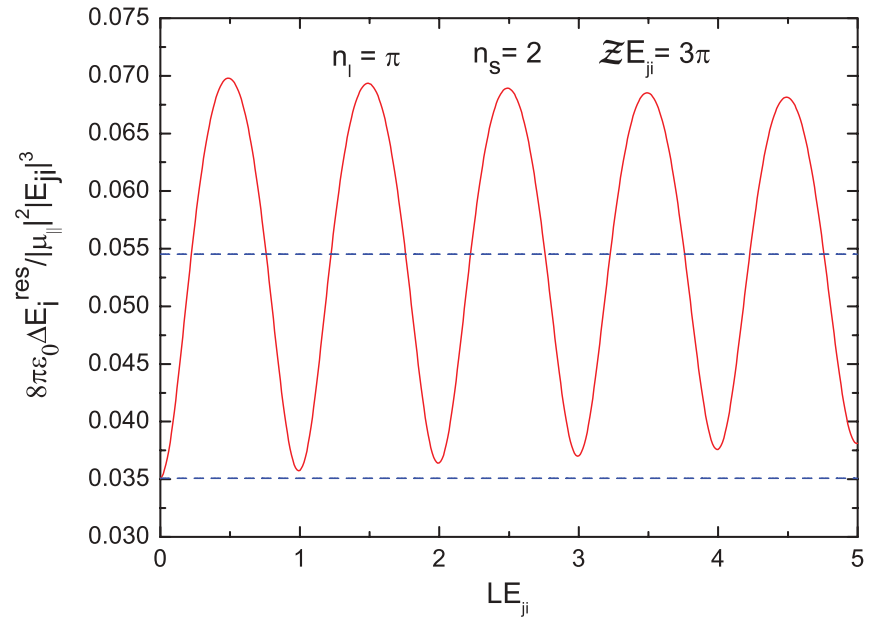

FIG. 15. (Color online) Plot of the exact energy-level shift (61) (resonant part) in an excited atom due to the parallel component of the atomic dipole moment placed in front of the layered dielectric with parameters as shown on the graph (solid line). The dashed lines represent energy shifts caused by the single half spaces of refractive index $n_{1}=2 \pi$ (top) and $n_{\mathrm{s}}=2$ (bottom).

less pronounced as the value of $L E_{j i}$ increases. This is because as we increase $L E_{j i}$ the resonances and antiresonances move closer and closer together so that their effects cancel out. It is interesting to note that this behavior could not have been inferred from Eq. (76), which indicates that the approximation (76) can be useful only for $L E_{j i} \ll 1$, which can also be easily verified numerically.

\section{SUMMARY}

Using perturbation theory we have calculated the energylevel shift in a neutral atom placed in front of a layered dielectric half-space, as shown in Fig. 1. The major difficulty in working out the energy shift is the sum over all modes that appears in this type of calculation [Eq. (50)], especially when the spectrum of the modes consists of the continuous and discrete parts (Secs. II A and II B). This obstacle can be circumvented by using complex-variable techniques to express the sum over all modes as a single contour integral in the complex $k_{z}$ plane [Eq. (54) and Fig. 4]. Then the energy shift (58) is easily analyzed asymptotically as well as numerically. For a ground-state atom, regardless of whether in retarded or nonretarded regimes, we find that the leading-order correction to the interaction of an atom with an unlayered interface is proportional to $L / \mathcal{Z}$. The asymptotic series are given by (67) and (72) and provide reasonable estimate of the influence of the single dielectric layer on the standard half-space result (Fig. 7). In the opposite case of a very thick layer, that is, $\mathcal{Z} / L \ll 1$, we find that the result is well approximated by a dielectric half space [9]. For excited atoms we find that the interaction between an atom and the layered dielectric (61) is subject to resonances that occur between the wavelength of the dominant atomic transition $\lambda_{j i}$ and the thickness of the layer $L$ (Sec. IV D). In particular, the interaction between an atom and the slab can be strongly suppressed in the retarded regime (cf. Fig. 11) whenever the optical thickness of the slab $\tau$ is equal to the half-integer multiple of the wavelength of the dominant atomic transition $\lambda_{j i}$. The existence of resonance effects suggests a physical picture of the excited atom as a radiating dipole. The resonance and antiresonance correspond to constructive and destructive interference. We have also provided reasonable approximations in the nonretarded (75) and retarded (76) regimes that can be used to quickly estimate the magnitude of the resonant interaction between an atom and a layered dielectric.

\section{APPENDIX A: FRESNEL COEFFICIENTS FOR LAYERED DIELECTRIC}

Here we list the reflection and transmission coefficients appearing in the normal modes of the system as discussed in the Sec. II A. For the left-incident modes we find

$$
\begin{aligned}
& R_{\lambda}^{L}=\frac{r_{\lambda}^{\mathrm{sl}}+r_{\lambda}^{\mathrm{lv}} e^{2 i k_{z 1} L}}{1+r_{\lambda}^{\mathrm{sl}} r_{\lambda}^{\mathrm{lv}} e^{2 i k_{z 1} L}} e^{-i k_{z \mathrm{~s}} L}, \\
& I_{\lambda}^{L}=\frac{t_{\lambda}^{\mathrm{sl}} e^{i\left(k_{z l}-k_{z \mathrm{~s}}\right) L / 2}}{1+r_{\lambda}^{\mathrm{sl}} r_{\lambda}^{\mathrm{lv}} e^{2 i k_{z l} L},} \\
& J_{\lambda}^{L}=\frac{t_{\lambda}^{\mathrm{sl}} r_{\lambda}^{\mathrm{lv}} e^{\left(3 i k_{z 1}-i k_{z \mathrm{~s}}\right) L / 2}}{1+r_{\lambda}^{\mathrm{sl}} r_{\lambda}^{\mathrm{lv}} e^{2 i k_{z 1} L}}, \\
& T_{\lambda}^{L}=\frac{t_{\lambda}^{\mathrm{s}} t_{\lambda}^{1 \mathrm{v}} e^{\left(2 i k_{z 1}-i k_{z \mathrm{~s}}-i k_{z}\right) L / 2}}{1+r_{\lambda}^{\mathrm{sl}} r_{\lambda}^{\mathrm{lv}} e^{2 i k_{z 1} L}},
\end{aligned}
$$

and for the right-incident modes we get

$$
\begin{gathered}
R_{\lambda}^{R}=\frac{r_{\lambda}^{\mathrm{vl}}+r_{\lambda}^{\mathrm{ls}} e^{2 i k_{z 1} L}}{1+r_{\lambda}^{\mathrm{vl}} r_{\lambda}^{\mathrm{ls}} e^{2 i k_{z} L} e^{-i k_{z} L},} \\
I_{\lambda}^{R}=\frac{t_{\lambda}^{\mathrm{vl}} e^{i\left(k_{z 1}-k_{z}\right) L / 2}}{1+r_{\lambda}^{\mathrm{vl}} r_{\lambda}^{\mathrm{s}} e^{2 i k_{z 1} L}}, \\
J_{\lambda}^{R}=\frac{t_{\lambda}^{\mathrm{vl}} r_{\lambda}^{\mathrm{ss}} e^{\left(3 i k_{z 1}-i k_{z}\right) L / 2}}{1+r_{\lambda}^{\mathrm{vl}} r_{\lambda}^{\mathrm{ls}} e^{2 i k_{z 1} L}}, \\
T_{\lambda}^{R}=\frac{t_{\lambda}^{\mathrm{vl}} t_{\lambda}^{1 \mathrm{~s}} e^{\left(2 i k_{z 1}-i k_{z \mathrm{~s}}-i k_{z}\right) L / 2}}{1+r_{\lambda}^{\mathrm{vl}} r_{\lambda}^{\mathrm{ls}} e^{2 i k_{z 1} L}} .
\end{gathered}
$$

The Fresnel reflection coefficients $r_{\lambda}^{\mathrm{ab}}$ for a single interface are given by (11).

\section{APPENDIX B: ELECTROSTATIC CALCULATION OF THE ENERGY-LEVEL SHIFT IN A GROUND-STATE ATOM IN A LAYERED GEOMETRY}

To provide an additional check on the consistency of our calculations we derive Eq. (66) by means of electrostatics. We start from the general formula derived in [20] that expresses the electrostatic interaction energy of a electric dipole in the presence of a dielectric in terms of Green's function of the Laplace equation

$$
\Delta E=\frac{1}{2} \sum_{i}\left\langle\mu_{i}^{2}\left|\nabla_{i} \nabla_{i}^{\prime} G_{H}\left(\mathbf{r}, \mathbf{r}^{\prime}\right)\right|_{\mathbf{r}=\mathbf{r}_{0}, \mathbf{r}^{\prime}=\mathbf{r}_{0}} .\right.
$$

Here the sum runs over three components of the dipole moment and the subscript $H$ means that only the homogeneous correction to the free-space Green's function that is caused by the presence of the boundary enters the formula. This ensures 
that the self-energy of the dipole is omitted and guarantees the convergence of the final result. The harmonic function $G_{H}\left(\mathbf{r}, \mathbf{r}^{\prime}\right)$ is a solution of the Laplace equation that vanishes for $|z| \rightarrow \infty$. Therefore, it can be written in the form

$$
\begin{aligned}
G_{H}\left(\mathbf{r}, \mathbf{r}^{\prime}\right) & \\
= & -\frac{1}{4 \pi \epsilon_{0}} \int_{0}^{\infty} d_{2} \mathbf{k}_{\|} e^{i \mathbf{k}_{\|} \cdot \mathbf{r}_{\|}} \\
& \times\left\{\begin{array}{cc}
C_{1}\left(\mathbf{k}_{\|}, \mathbf{r}^{\prime}\right) e^{k_{z} z}, & z<L / 2, \\
C_{2}\left(\mathbf{k}_{\|}, \mathbf{r}^{\prime}\right) e^{k_{z} z}+C_{3}\left(\mathbf{k}_{\|}, \mathbf{r}^{\prime}\right) e^{-k_{z} z}, & |z|<L / 2, \\
C_{4}\left(\mathbf{k}_{\|}, \mathbf{r}^{\prime}\right) e^{-k_{z} z}, & z>L / 2,
\end{array}\right.
\end{aligned}
$$

with $k_{z}=\sqrt{k_{x}^{2}+k_{y}^{2}}$. The $C$ coefficients are easily worked out by applying the continuity conditions, which result from Maxwell's equations, across the interfaces and one finds that

$$
\begin{aligned}
G_{H}\left(\mathbf{r}, \mathbf{r}^{\prime}\right)= & -\frac{1}{4 \pi \epsilon_{0}} \int_{0}^{\infty} d k J_{0}(k \rho) e^{-k\left(z+z^{\prime}\right)} \\
& \times \frac{\frac{n_{1}^{2}-1}{n_{1}^{2}+1}-\frac{n_{1}^{2}-n_{\mathrm{s}}^{2}}{n_{\mathrm{s}}^{2}+n_{1}^{2}} e^{-2 k L}}{1-\frac{n_{1}^{2}-1}{n_{1}^{2}+1} \frac{n_{1}^{2}-n_{\mathrm{s}}^{2}}{n_{\mathrm{s}}^{2}+n_{1}^{2}} e^{-2 k L}}
\end{aligned}
$$

with $\rho=\sqrt{\left(x-x^{\prime}\right)^{2}+\left(y-y^{\prime}\right)^{2}}$. Application of the formula (B1) is straightforward and we easily derive that the electrostatic interaction energy of a dipole in a vicinity of the layered dielectric is indeed equal to (66).

\section{APPENDIX C: RETARDED LIMIT OF THE INTERACTION ENERGY BETWEEN AN ATOM AND A DIELECTRIC HALF SPACE}

The interaction between an atom and a nondispersive dielectric half space has been considered in detail in [9]. It has been shown there that, to leading-order, the energy shift in the retarded limit can be expressed as

$$
\Delta E_{n_{\mathrm{s}}}^{\mathrm{ret}}=-\frac{3}{64 \pi^{2} \epsilon_{0} \mathcal{Z}^{4}} \sum_{j \neq i}\left(\frac{c_{\|}\left|\mu_{\|}\right|^{2}+c_{\perp}\left|\mu_{\perp}\right|^{2}}{E_{j i}}\right),
$$

with the coefficients $c_{\|, \perp}$ given by

$$
\begin{aligned}
c_{\|}= & -\frac{1}{n_{\mathrm{s}}^{2}-1}\left(\frac{2}{3} n_{\mathrm{s}}^{2}+n_{\mathrm{s}}-\frac{8}{3}\right) \\
& +\frac{2 n_{\mathrm{s}}^{4}}{\left(n_{\mathrm{s}}^{2}-1\right) \sqrt{n_{\mathrm{s}}^{2}+1}} \ln \left(\frac{\sqrt{n_{\mathrm{s}}^{2}+1}+1}{n_{\mathrm{s}}\left[\sqrt{n_{\mathrm{s}}^{2}+1}+n_{\mathrm{s}}\right]}\right) \\
& +\frac{2 n_{\mathrm{s}}^{4}-2 n_{\mathrm{s}}^{2}-1}{\left(n_{\mathrm{s}}^{2}-1\right)^{3 / 2}} \ln \left(\sqrt{n_{\mathrm{s}}^{2}+1}+n_{\mathrm{s}}\right), \\
c_{\perp}= & \frac{1}{n_{\mathrm{s}}^{2}-1}\left(4 n_{\mathrm{s}}^{4}-2 n_{\mathrm{s}}^{3}-\frac{4}{3} n_{\mathrm{s}}^{2}+\frac{4}{3}\right) \\
& -\frac{4 n_{\mathrm{s}}^{6}}{\left(n_{\mathrm{s}}^{2}-1\right) \sqrt{n_{\mathrm{s}}^{2}+1}} \ln \left(\frac{\sqrt{n_{\mathrm{s}}^{2}+1}+1}{n_{\mathrm{s}}\left[\sqrt{n_{\mathrm{s}}^{2}+1}+n_{\mathrm{s}}\right]}\right) \\
& -\frac{2 n_{\mathrm{s}}^{2}\left(2 n_{\mathrm{s}}^{4}-2 n_{\mathrm{s}}^{2}+1\right)}{\left(n_{\mathrm{s}}^{2}-1\right)^{3 / 2}} \ln \left(\sqrt{n_{\mathrm{s}}^{2}-1}+n_{\mathrm{s}}\right) .
\end{aligned}
$$

[1] A. Contreras Reyes and C. Eberlein, Phys. Rev. A 80, 032901 (2009).

[2] J. M. Wylie and J. E. Sipe, Phys. Rev. A 32, 2030 (1985).

[3] S. Y. Buhmann and D.-G. Welsh, Prog. Quantum Electron. 31, 51 (2007).

[4] A. D. McLachlan, Proc. R. Soc. London A 271, 387 (1963).

[5] S. Y. Buhmann, L. Knoll, D.-G. Welsch, and H. Dung, Phys. Rev. A 70, 052117 (2004).

[6] J. M. Wylie and J. E. Sipe, Phys. Rev. A 30, 1185 (1984).

[7] C. K. Carnigila and L. Mandel, Phys. Rev. D 3, 280 (1971).

[8] H. Koshravi and R. Loudon, Proc. R. Soc. London A 433, 337 (1991); 436, 373 (1992).

[9] S. Wu and C. Eberlein, Proc. R. Soc. London, Ser. A 455, 2487 (1999).

[10] E. Yablonovitch, T. J. Gmitter, and R. Bhat, Phys. Rev. Lett. 61, 2546 (1988).

[11] M. Bordag, Phys. Rev. D 70, 085010 (2004); 76, 065011 (2007).
[12] R. J. Glauber and M. Levenstein, Phys. Rev. A 43, 467 (1991).

[13] J. D. Jackson, Classical Electrodynamics (Wiley, New York, 1962).

[14] R. Zietal, Quantum Electrodynamics near material boundaries, Ph.D. thesis, University of Sussex, Brighton, 2010 (unpublished).

[15] M. Abramowitz and I. Stegun, eds., Handbook of Mathematical Functions (US GPO, Washington, DC, 1964).

[16] H. P. Urbach and G. L. J. A. Rikken, Phys. Rev. A 57, 3913 (1998).

[17] A. M. Contreras Reyes and C. Eberlein, Phys. Rev. A 79, 043834 (2009).

[18] I. Bialynicki-Birula and J. B. Brojan, Phys. Rev. D 5, 485 (1972).

[19] C. Eberlein and D. Robaschik, Phys. Rev. D 73, 025009 (2006).

[20] C. Eberlein and R. Zietal, Phys. Rev. A 75, 032516 (2007).

[21] C. M. Bender and S. A. Orszag, Advanced Mathematical Methods for Scientists and Engineers (Springer Science + Business Media, New York, 1999). 